\title{
SPIDERS FOR RANK 2 LIE ALGEBRAS
}

\author{
GREG KUPERBERG
}

\begin{abstract}
A spider is an axiomatization of the representation theory of a group, quantum group, Lie algebra, or other group or group-like object. It is also known as a spherical category, or a strict, monoidal category with a few extra properties, or by several other names. A recently useful point of view, developed by other authors, of the representation theory of $\operatorname{sl}(2)$ has been to present it as a spider by generators and relations. That is, one has an algebraic spider, defined by invariants of linear representations, and one identifies it as isomorphic to a combinatorial spider, given by generators and relations. We generalize this approach to the rank 2 simple Lie algebras, namely $A_{2}, B_{2}$, and $G_{2}$. Our combinatorial rank 2 spiders yield bases for invariant spaces which are probably related to Lusztig's canonical bases, and they are useful for computing quantities such as generalized $6 j$-symbols and quantum link invariants. Their definition originates in definitions of the rank 2 quantum link invariants that were discovered independently by the author and Francois Jaeger.
\end{abstract}

\section{INTRODUCTION}

One of the problems of classical invariant theory is to characterize, for all $n$-tuples $V_{1}, \ldots, V_{n}$ of finitedimensional, irreducible representations over $\mathbb{C}$ of a compact group $G$ or simple Lie algebra $\mathfrak{g}$, the vector space of multilinear functions

$$
f: V_{1} \times V_{2} \times \ldots \times V_{n} \rightarrow \mathbb{C}
$$

which are invariant under the action of $G$ or $\mathfrak{g}$. In more modern terminology, the problem is to characterize the dual vector space of invariant tensors

$$
\operatorname{Inv}\left(V_{1} \otimes V_{2} \otimes \ldots \otimes V_{n}\right),
$$

or just $\operatorname{Inv}(V)$ if $V$ is a tensor product of irreducibles. (Also, instead of working over $\mathbb{C}$, one might work over some other field $\mathbb{F}$ of characteristic 0.) Of course, for a simple Lie algebra $\mathfrak{g}$, the dimension of such a vector space is given by Cartan-Weyl character theory. But it is also useful to consider operations on invariant spaces such as tensor products and contractions. Even for finite-dimensional, simple Lie algebras over $\mathbb{C}$, these operations are not completely understood.

Interest in multilinear invariant theory was revived after the discovery of quantum groups. A quantum Lie group is a non-commutative, non-cocommutative Hopf algebra $U_{q}(\mathfrak{g})$ which is a deformation of the universal enveloping algebra $U(\mathfrak{g})$. In fact, the reprentation theory of a quantum group is just as important than the quantum group itself and may also be defined using loop groups or conformal quantum field theory. Among other uses of quantum groups, a quantum invariant of a tangle takes is a vector in an invariant $\operatorname{space} \operatorname{Inv}(V)$ where $V$ depends on the boundary of the tangle and other data.

Because of non-cocommutativity, the switching map

$$
\tau: V \otimes W \rightarrow W \otimes V
$$

given by

$$
x \otimes y \mapsto y \otimes x
$$

is in general not an (equivariant) map between quantum group representations. Thus, there is no natural symmetric group action on invariant spaces with $n$ tensor factors with $n>2$. (There is often a braid group action.) However, the following operations exist and are natural:

1.: Tensor product:

$$
\operatorname{Inv}(V) \otimes \operatorname{Inv}(W) \hookrightarrow \operatorname{Inv}(V \otimes W)
$$

1991 Mathematics Subject Classification. Primary 17B37; Secondary 81R50, 57M25.

The author was supported by an NSF Postdoctoral Fellowship, grant \#DMS-9107908. 
2.: Cyclic permutation:

$$
\operatorname{Inv}(V \otimes W) \rightarrow \operatorname{Inv}(W \otimes V)
$$

3.: Contraction:

$$
\operatorname{Inv}\left(V \otimes V^{*} \otimes W\right) \rightarrow \operatorname{Inv}(W)
$$

Since $V$ and $W$ may themselves be tensor produts, cyclic permutation of two tensor factors yields cyclic permutation of $n$ tensor factors, but not general permutations. Also, contraction must be interpreted carefully, because in a quantum group, one must reverse order when taking duals: $(V \otimes W)^{*} \cong W^{*} \otimes V^{*}$.

A spider is an abstraction of a representation theory with these three operations. It is a collection of vector spaces, or perhaps modules or sets, to be thought of as invariant spaces, together with abstract operations called join, rotation, and stitch, to be thought of as tensor product, rotation, and contraction. It is both convenient and conceptually important to depict these operations with certain planar graphs. These graphs are called webs, hence the term "spider".

Another motivation of the spider operations is that they really describe the entire equivariant tensor category of representations of a group, Lie algebra, or quantum group. In general,

$$
\operatorname{Hom}(A, B) \cong \operatorname{Inv}\left(A^{*} \otimes B\right)
$$

the tensor product of two homomorphisms can be defined in terms of tensor product and cyclic permutation of invariants, and composition of homomorphisms can be defined in terms of tensor product and contraction. Contrariwise, the spider operations can be defined in terms of tensor product and composition of morphisms. For this reason, spiders are sometimes defined as a type of (non-symmetric) tensor category. To construct such a category, one must divide the tensor factors of an invariant space

$$
\operatorname{Inv}\left(V_{1} \otimes \ldots \otimes V_{n}\right)
$$

into smaller tensor products which serve as the domain and target of a space of morphisms. This involves arbitrary choices that are extraneous to most of the arguments in this paper, so we will not usually treat spiders as categories.

In this paper, we will define certain spiders in terms of generators and relations, and we will show that they are isomorphic to the representation theories of rank two Lie algebras and the quantum deformations of these representation theories. These results generalize a well-known construction for $A_{1}=\operatorname{sl}(2)$ that first arose in a paper of Rumer, Teller, and Weyl [18], that was developed later by Temperley and Lieb [22], and that was greatly developed recently by Jones, Kauffman, Lickorish, Masbaum, and Vogel [5, 6, 10, 12]. Moreover, Frenkel and Khovanov have recently established that the bases of invariant spaces that arise when one constructs the $A_{1}$ spider by generators and relations are dual to the canonical bases of Lusztig [11]. We conjecture that a similar phenomenon holds in the rank 2 cases.

1.1. Acknowledgements. The author would like to thank Sarah Witherspoon, Bruce Westbury, Igor Frenkel, and Mikhail Khovanov for their attention to this paper and the work it presents. Also, the TEX macro package PSTricks [23] was essential for typesetting the equations and figures.

\section{THE $A_{1}$ SPIDER AND $\operatorname{sl}(2, \mathbb{F})$}

Let $V \cong \mathbb{F}^{2}$ be the defining representation of $\operatorname{sl}(2, \mathbb{F})$, where $\mathbb{F}$ is some field of characteristic 0 . (The complex numbers $\mathbb{C}$ are a good choice for $\mathbb{F}$.) Choose a parameter $a \in \mathbb{F}$. Since the vector space $V$ is a self-dual representation, there exists a non-degenerate, invariant contraction operation $\sigma: V \otimes V \rightarrow \mathbb{F}$. For each $n$, choose $n$ points on the boundary of a disk in the plane, and let $B_{n}$, the set of basis webs, be the set of crossingless matchings of the $n$ points. For example, if $n$ is odd, $B_{n}$ is empty, while $B_{6}$ has the following 5 elements:
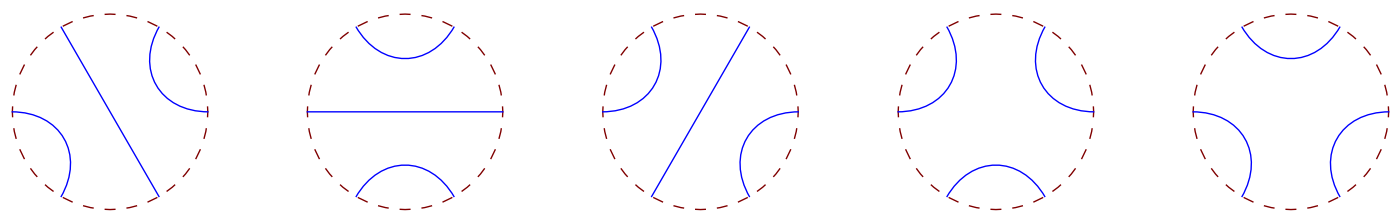

By convention, $B_{0}$ has a single element, the empty disk. Let $W_{n}$, the web space, be the vector space of formal linear combinations of elements of $B_{n}$ with coefficients in $\mathbb{F}$. 
Theorem 2.1. There exist isomorphisms $\phi_{n}: W_{n} \rightarrow \operatorname{Inv}\left(V^{\otimes n}\right)$.

Theorem 2.1 has few consequences in isolation; it only says that web spaces have the same dimension as invariant spaces. By the Weyl character formula, $\operatorname{dim} \operatorname{Inv}\left(V^{\otimes n}\right)$ is the number of non-negative lattice paths of length $n$ in one dimension, and there is a standard combinatorial bijection between such paths and crossingless matchings. (Idea: Both combinatorial sets are equivalent to balanced lists of parentheses of length $n$, such as " $(())$ ()" when $n=6$.)

A rotation operation is the linear extension to a web space of rotation of basis webs:

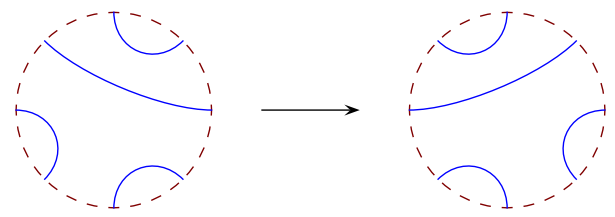

A join operation is a bilinear operation on a pair of web spaces. Given two basis webs, their join is given by connecting their disks by a band:

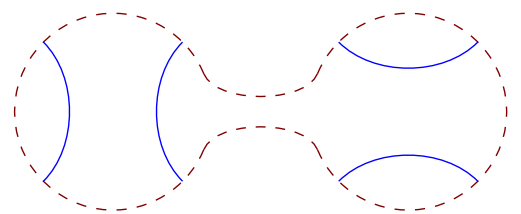

A join operation extends bilinearly to arbitrary webs. Finally, a stitch operation is a linear transformation between web spaces. Given a basis web, its stitch at an adjacent pair of vertices is given by connecting the vertices by an arc:

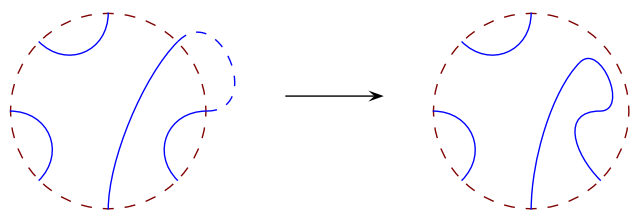

If the result produces a closed loop, then strictly speaking, it is not a matching of vertices on the boundary and therefore it is not a basis web. In this case, erase the closed loop and replace it by a factor of $a$, the element of $\mathbb{F}$ chosen at the beginning:

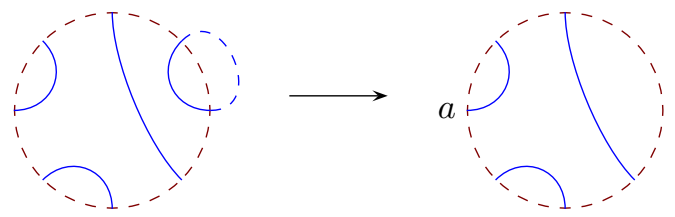

The factor of $a$ is a reminder that stitch is a linear operation whose value on a basis web might be a non-basis web. A stitch also extends linearly from a family of map $B_{n} \rightarrow W_{n-2}$ to a map $W_{n} \rightarrow W_{n-2}$.

The combinatorial $A_{1}$ spider, parameterized by $a$, is the list of web spaces, together with all rotation, join, and stitch operations.

Theorem 2.2 (Rumer,Teller,Weyl). If $a=-2$, then the isomorphisms $\phi_{n}: W_{n} \rightarrow \operatorname{Inv}\left(V^{\otimes n}\right)$ can be uniquely chosen to send the operations of join to tensor product, stitch to contraction, and rotation to cyclic permutation of tensor factors composed with negation.

(Difference in sign between rotation and cyclic permutation of tensor factors is explained in Section 3.)

The conversion of join to tensor product requires some explanation. Tensor products depend on a linear ordering of tensor factors, but the vertices in a web are only cyclically ordered. To realize an explicit association between webs and invariant tensors, it is necessary to refine the cyclic ordering to a linear ordering. Then join or tensor product becomes a process of concatenation:

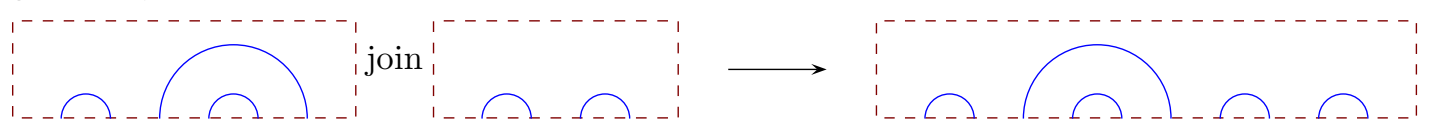


The general operation of join corresponds to tensor product modified by cyclic permutations.

We explictly construct the $\phi_{n}$ 's to demonstrate their uniqueness. Assume some non-zero value $r \in V \otimes V$ for $\phi_{2}$ of a line segment:

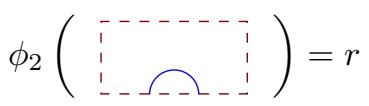

Any other basis web is obtained from a line segment by repeated joins. Therefore its image under $\phi_{n}$ is, up to sign, a combination of cyclic permutations and tensor products of $r$ 's. For example, $\phi_{4}$ of the basis web

while

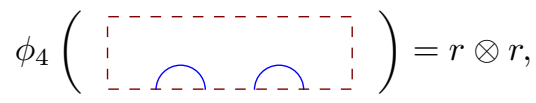

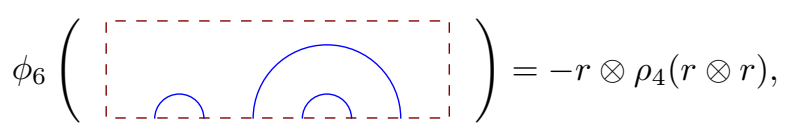

where $\rho_{4}$ is cyclic permutation of four tensor factors. It remains only to determine $r$. The stitch of two arcs is one arc:

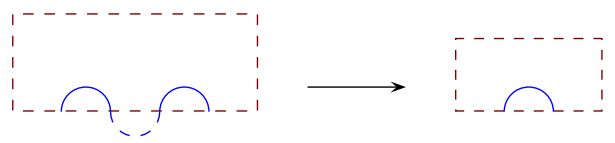

Applying $\phi_{4}$ and $\phi_{2}$ to the two sides yields

$$
(I \otimes \sigma \otimes I)(r \otimes r)=r .
$$

This equation says that $r$ is the inverse of $\sigma$ in an indirect sense. More explicitly, given a basis $\left\{e_{1}, e_{2}\right\}$ for $V$, one natural choice for $r$ and $\sigma$ is:

$$
\begin{gathered}
\sigma\left(e_{2} \otimes e_{1}\right)=-\sigma\left(e_{1} \otimes e_{2}\right)=1 \\
\sigma\left(e_{1} \otimes e_{1}\right)=\sigma\left(e_{2} \otimes e_{2}\right)=0 \\
r=e_{1} \otimes e_{2}-e_{2} \otimes e_{1}
\end{gathered}
$$

But in a more direct sense, $r$ is not the inverse of $\sigma$, because with this choice of $r$ and $\sigma$, as with every other compatible pair of choices, a closed loop, or $\sigma(r)$, is -2 .

Checking that the $\phi_{n}$ 's take spider operations to tensor operations reduces to comparing definitions and simple calculations. The least trivial part of Theorem 2.2 is the assertion that each $\phi_{n}$ is an isomorphism. Since the domain and target of $\phi_{n}$ have the same dimension, it suffices to establish surjectivity. To prove surjectivity, we use the isomorphism

$$
\operatorname{Inv}\left(V^{\otimes 2 n}\right) \cong \operatorname{End}\left(V^{\otimes n}\right)
$$

By the Fundamental Theorem of Invariant Theory of Schur and Weyl, the endomorphisms of $V^{\otimes n}$ are spanned by permutations of tensor factors. Such a permutation can be depicted by a diagram of matched dots:

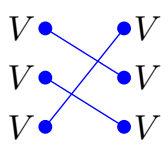

It is a composition of many copies of the switching map $\tau: x \otimes y \mapsto y \otimes x$ in $\operatorname{End}(V \otimes V)$. A calculation demonstrates that $\tau$ lies in the image of $\phi_{4}$ :

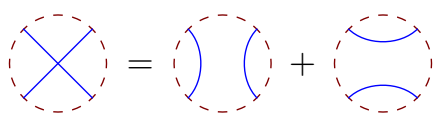

It follows by multilinear expansion that every permutation is in the image of $\phi_{2 n}$. Since the permutations span, $\phi_{2 n}$ is surjective.

What if $a$ is not -2 ? The specialization of the spider should describe the representation theory of some object related to $\operatorname{sl}(2, \mathbb{F})$. The quantum group $U_{q}(\operatorname{sl}(2, \mathbb{F}))$ was invented essentially for this purpose. One parameterization of the $A_{1}$ spider is $a=-q^{1 / 2}-q^{-1 / 2}$ over the field $\mathbb{C}\left(q, q^{1 / 2}, q^{1 / 3}, \ldots\right)$. (Note, however, that the spider is actually defined over the $\operatorname{ring} \mathbb{C}\left[q, q^{-1}, q^{1 / 2}, q^{1 / 3}, \ldots\right]$, which allows us to specialize to any value of $q \in \mathbb{C}^{*}$.) Another standard notation is $a=-[2]$, where, by definition,

$$
[n]=\frac{q^{n / 2}-q^{-n / 2}}{q^{1 / 2}-q^{-1 / 2}} .
$$


2.1. Computations with the $A_{1}$ spider. A basis web, or crossingless matching, is an equivalence class, modulo boundary-fixing isotopies, of proper embeddings in the disk of a 1-manifold with no circles. Although proper embeddings of loop-free 1-manifolds suffice to define the web space $W_{n}$, it is convenient to define any properly embedded 1-manifold in the disk as a web. Specifically, a 1-manifold with $n$ closed loops denotes the web which is $a^{n}$ times the web obtained by erasing all closed loops.

Given this meaning for all embedded 1-manifolds, basis webs such as

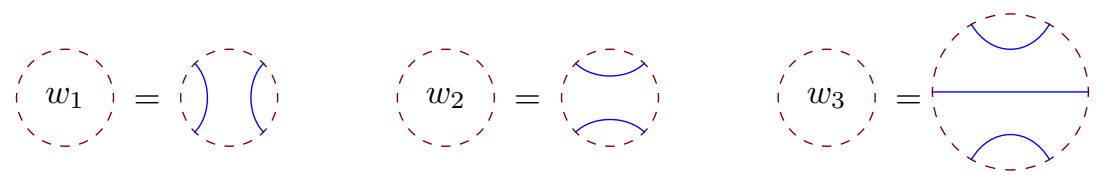

can be embedded in larger disks to form 1-manifolds which are therefore other webs:

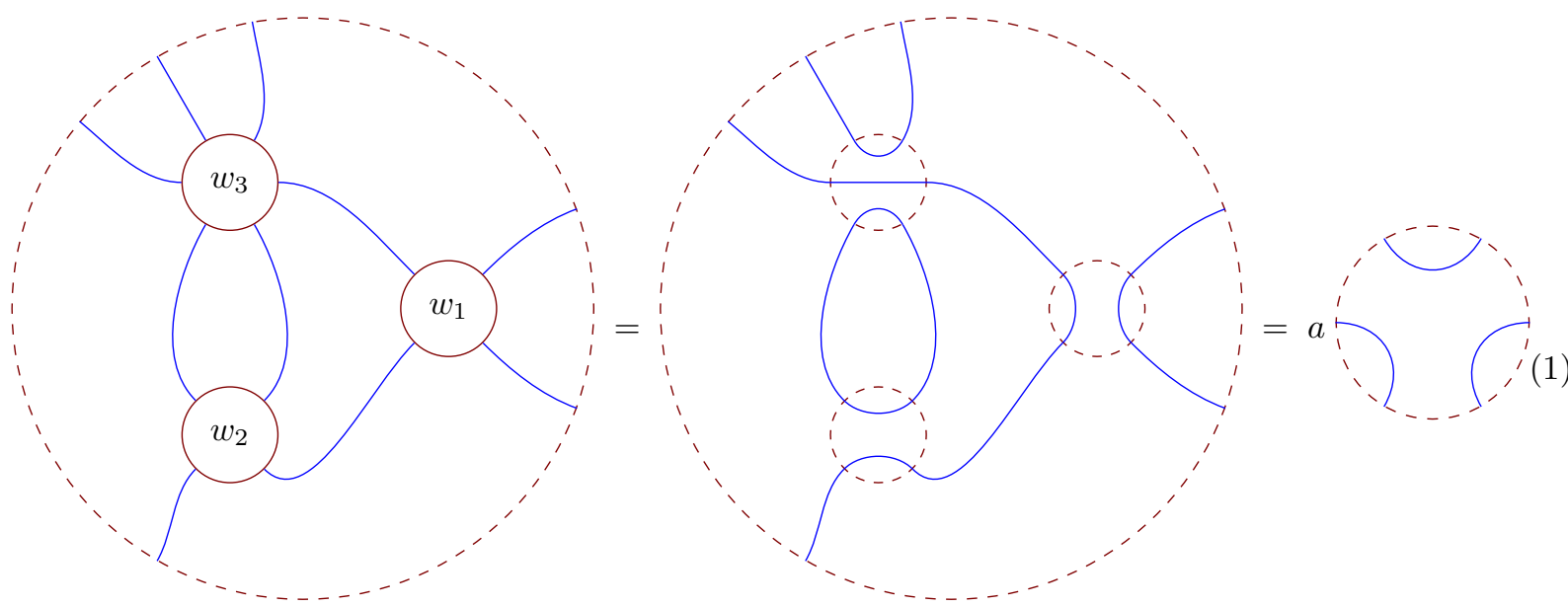

If the $w_{i}$ 's are arbitrary webs, then a diagram such as the one in equation (11) denotes a multilinear expansion and is called a compound web. For example, if

$$
\left.\begin{array}{l}
w \\
\cdots
\end{array}=2 i\right)^{\prime}(i+3 i
$$

then

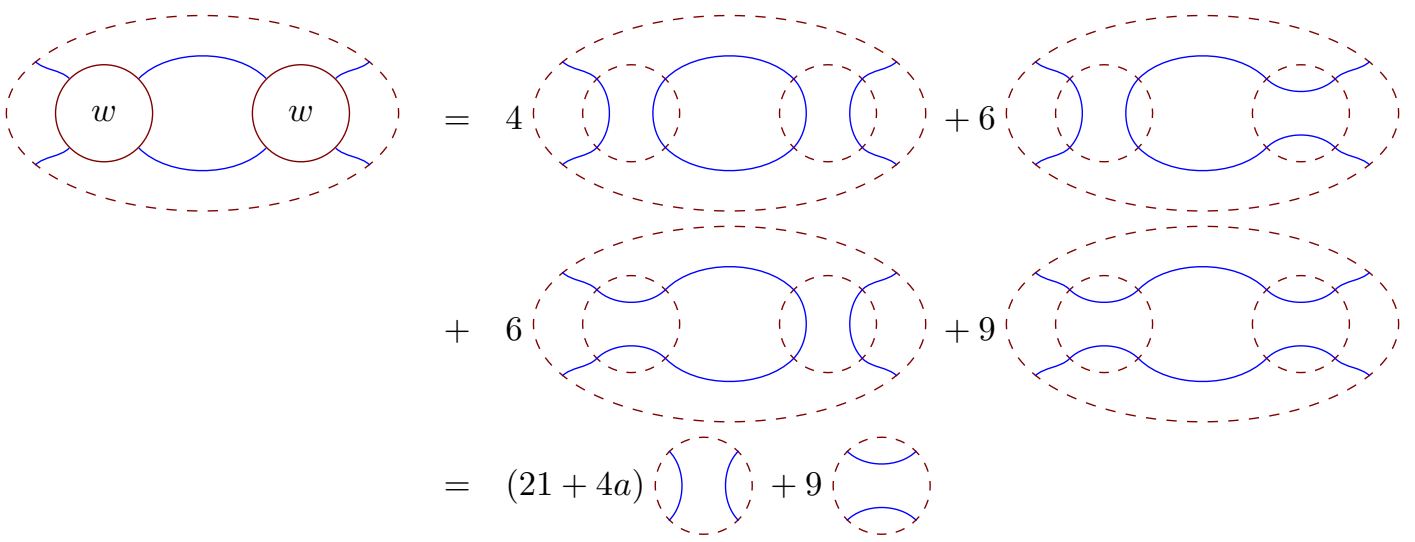

Another view of a compound web is that it is a sequence of joins and stitches of the component webs. It can be realized by many different such sequences, but they all have the same final value.

An important class of examples of compound webs are those generated by a web called a crossing:

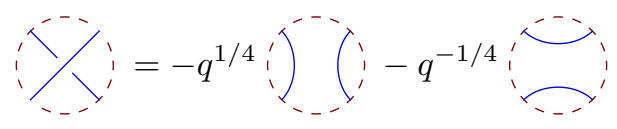


Given this definition, it is easy to check the identities
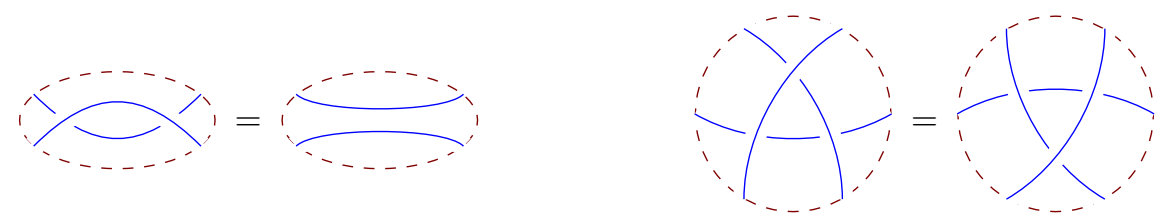

These are known as the second and third Reidemeister moves. In particular, a clever trick due to Kauffman [6], namely replacing one of the crossings in the third move by its linear expansion, reduces the third move to the second one. If we interpret a compound web made from copies of a crossing as a tangle or link projection, the identities are also known as the second and third Reidemeister moves. More generally, a projection of a tangle or link such as
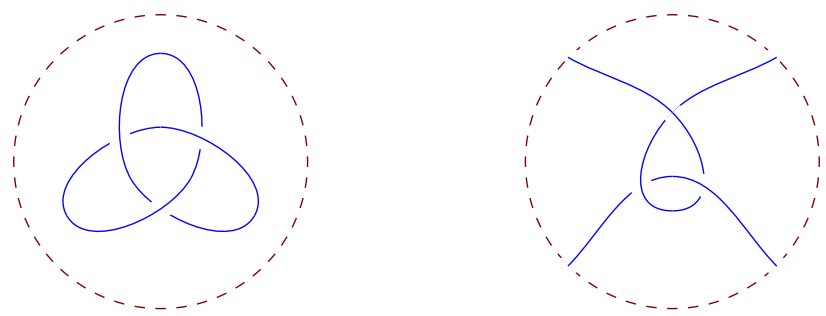

evaluates to a vector in some web space. The value of a link projection lies in $W_{0}$, a 1 -dimensional vector space with basis the empty web. Its single coefficient is a Laurent polynomial in $q^{1 / 4}$. Given invariance under the second and third Reidemeister moves, this function on link projections is a regular isotopy invariant. It is also covariant under full isotopy; it gains a factor of $q^{ \pm 3 / 4}$ under the first Reidemeister move:

$$
\mathcal{O}^{\cdots} !=q^{3 / 4} !{ }^{\prime}
$$

(Since $W_{2}$ is 1-dimensional, these two webs must be proportional.) This polynomial is known as the Kauffman bracket, and up to normalization it equals the Jones polynomial.

Another important type of compound web is a concatenation of two webs. Given a web in $W_{a+b}$, divide its endpoints into a segment of $a$ points and a segment of $b$ points. Given another web in $W_{b+c}$ whose vertices are divided into segments of length $b$ and $c$, their concatenation consists of connecting $b$ adjacent pairs:

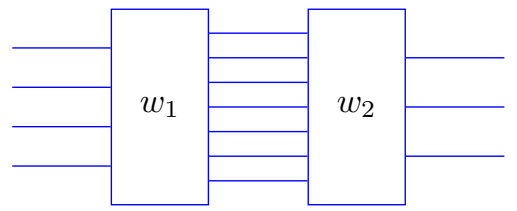

With concatenation operations, the $A_{1}$ spider can be understood as a category, isomorphic to a subcategory of the (quantum) representation category of $\mathrm{sl}(2, \mathbb{F})$, whose objects are segments of points and whose arrows are webs. For a fixed $n$, the endomorphisms of the object consisting of $n$ points form an associative algebra, called the Temperley-Lieb algebra. As a unital algebra, it is generated by $e_{1}, \ldots, e_{n-1}$, where $e_{i}$ is a basis web which is a pair of U-turns at the $i$ th and $i+1$ st positions:

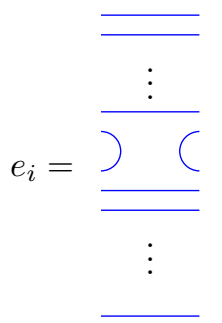


It is easy to show that

$$
\begin{aligned}
& e_{i} e_{j}=e_{j} e_{i}, \quad i \neq j \pm 1 \\
& e_{i} e_{i \pm 1} e_{i}=e_{i} \\
& e_{i}^{2}=-[2] e_{i}
\end{aligned}
$$

is a complete set of relations for the Temperley-Lieb algebra.

2.2. Other representations of $\operatorname{sl}(2, \mathbb{F})$. So far, we have only described $\operatorname{Inv}\left(V^{\otimes n}\right)$ and not

$$
\operatorname{Inv}\left(A_{1} \otimes A_{2} \otimes \ldots \otimes A_{k}\right)
$$

for arbitrary irreducible representations $A_{1}, \ldots, A_{k}$ of $\operatorname{sl}(2, \mathbb{F})$. The Lie algebra $\operatorname{sl}(2, \mathbb{F})$ has an irreducible representation $V_{n}$ of dimension $n+1$ for every non-negative $n$, and any finite-dimensional irreducible is isomorphic to one of these. The representation $V_{n}$ can be viewed as the $n$th symmetric power of $V$, in which case the equivariant contraction $\sigma$ induces an equivariant contraction

$$
\sigma_{n}: V_{n} \otimes V_{n} \rightarrow \mathbb{C}
$$

It is determined by the rule that

$$
\sigma_{n}\left(v^{n} \otimes w^{n}\right)=\sigma(v \otimes w)^{n} .
$$

Given positive integers $n_{1}, \ldots, n_{k}$ with sum $n$, consider a circle with $n$ distinguished points, partitioned into consecutive strings of points of length $n_{1}, n_{2}, \ldots, n_{k}$. Each string of points is called an external clasp. The clasped web space $W\left(n_{1}, \ldots, n_{k}\right)$ is a vector subspace of the web space $W_{n}$, defined as the span of those basis webs of with no U-turns between two endpoints in the same clasp. The set of such basis webs is denoted $B\left(n_{1}, \ldots, n_{k}\right)$. For example, the web

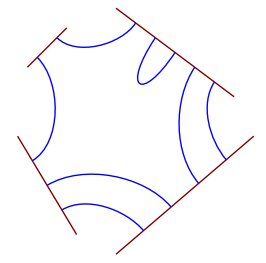

is not a basis web of $W(2,3,4,5)$, which instead has basis
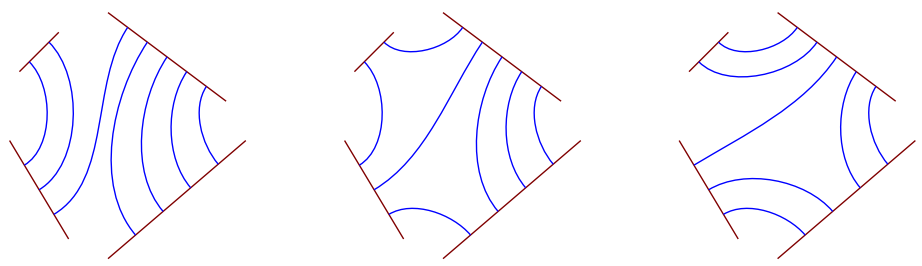

Like a web with closed loops, a clasped web with U-turns has a meaning as a web, but not a basis web. If there are any U-turns, the clasped web is defined to be the zero web. Thus, $W\left(n_{1}, \ldots, n_{k}\right)$ is also a quotient space of $W_{n}$.

Theorem 2.3. Let $N=\left(n_{1}, \ldots, n_{k}\right)$ be a multi-index, with

$$
V_{N}=V_{n_{1}} \otimes \ldots \otimes V_{n_{k}} .
$$

When $q=1$, there is a family of vector space isomorphisms

$$
\phi_{N}: W(N) \rightarrow \operatorname{Inv}\left(V_{N}\right)
$$

that send join to tensor product, stitch to contraction, and rotation to cyclic permutation of tensor factors up to sign.

Rotation and join of clasped webs are defined in the same way as for unclasped webs, but stitch is more complicated. Given a clasped web with two adjacent external clasps of the same size, the stitch of the web 
is obtained by identifying the two clasps and introducing an internal clasp, usually depicted as a box:

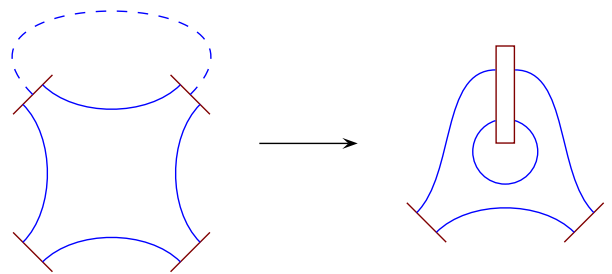

An internal clasp of size $n$ is a particular web in $W_{2 n}$ that satisfies the axioms

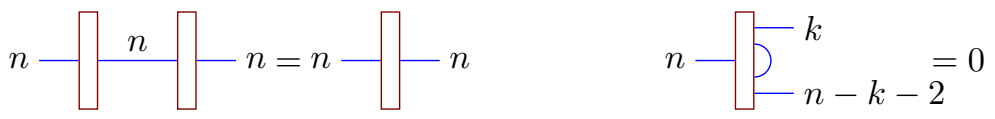

Equation (2.2) uses the convention that a strand labelled by $n$ denotes $n$ parallel strands. The equation says that an internal clasp is an idempotent of the $n$-strand Temperley-Lieb algebra and it annihilates all basis webs other than the identity on the right. Therefore a clasp concatenated with any web is proportional to a clasp. In particular, a clasp is unique, if it exists, and it has the same annihilation property on the left. The Wenzl recursion formula [24] demonstrates that clasps do exist, at least for most values of $q$ :

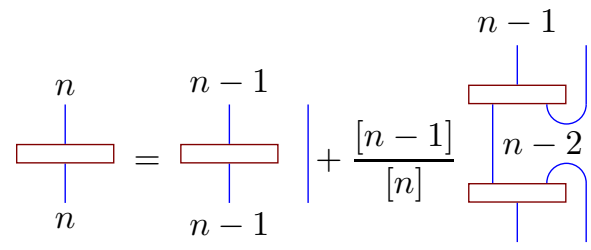

$n-1$

Internal clasps are also called magic weaving elements [7], boxes [10, and Jones-Wenzl idempotents [12].

An internal clasp is the concatenation of the unique basis web of $W(n, 1,1,1, \ldots, 1)$ with itself:

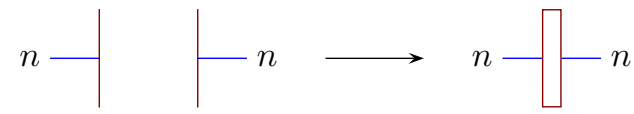

In terms of representations, this concatenation is a composition of the form

$$
V^{\otimes n} \rightarrow V_{n} \rightarrow V^{\otimes n}
$$

Since the composition is a non-zero idempotent, it is the equivariant projection from $V^{\otimes n}$ to its highestweight irreducible summand.

2.3. Isomorphism and equinumeration. In this section, we prove Theorem 2.3. It is fairly easy to construct each $\phi_{N}$ and to prove that is surjective. If $n=n_{1}+\ldots+n_{k}$, there is a projection

$$
\pi_{N}=\operatorname{Inv}\left(V^{\otimes n}\right) \rightarrow \operatorname{Inv}\left(V_{N}\right),
$$

and let $\phi_{N}=\pi_{N} \circ \phi_{n} \circ i_{N}$, where $i_{N}$ is the inclusion $W(N) \subset W_{n}$. Suppose that the $i$ th clasp of a web $w$ has $k$ U-turns, and let $m=n-2 k$. Then the invariant $\pi_{N} \circ \phi_{n}(w)$ lies in the image of some map which has a tensor factor of the form $V^{\otimes m} \rightarrow V_{n_{i}}$, where $m<n_{i}$, and any such map must be zero.

Thus, $\pi_{N} \circ \phi_{n}=\phi_{N} \circ j_{N}$, where $j_{N}$ is the projection $W_{n} \rightarrow W(N)$. From this, it is routine to show that the maps $p h i_{N}$ take spider operations to spider operations. Moreover, each $\phi_{N}$ is surjective, because both $\pi_{N}$ and $\phi_{n}$ are surjective and $i_{n}$ complements the kernel of $\pi_{N} \circ \phi_{n}$.

We complete the proof that $\phi_{N}$ is an isomorphism by demonstrating that its domain and target have the same dimension, thereby generalizing Theorem 2.1 to the clasped case:

Theorem 2.4. If $N$ is a multi-index, then

$$
\operatorname{dim} W(N)=\operatorname{dim} \operatorname{Inv}\left(V_{N}\right) .
$$

Proof: The proof is by induction on $|N|$, the number of indicies in $N$. The relation is straightforward for $|N| \leq 2$, so we first assume that $|N|=3$.

The Clebsch-Gordan theorem states that

$$
\operatorname{dim} \operatorname{Inv}\left(V_{i} \otimes V_{j} \otimes V_{k}\right)=1
$$


if each of $n, m$, and $l$ is less than or equal to the sum of the other two and if the sum of all three is even, and

$$
\operatorname{dim} \operatorname{Inv}\left(V_{i} \otimes V_{j} \otimes V_{k}\right)=0
$$

otherwise. On the other hand, $B(i, j, k)$ has at most one element:

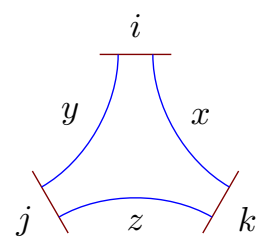

This web exists when there are non-negative integers $x, y$, and $z$ such that $i=x+y, j=x+z$, and $k=y+z$. These two conditions on $i, j$, and $k$ are equivalent.

Now suppose that $|N|>3$ and express the multi-index $N$ as $J K$, where $J$ and $K$ each have length at least 2. Since the $V_{n}$ 's constitute all finite-dimensional irreducible representations, and since they are self-dual, there is a decomposition

$$
\operatorname{Inv}\left(V_{J} \otimes V_{K}\right) \cong \bigoplus_{\ell} \operatorname{Inv}\left(V_{J} \otimes V_{\ell}\right) \otimes \operatorname{Inv}\left(V_{\ell} \otimes V_{K}\right)
$$

where $\ell$ is a single index rather than a multi-index. It suffices to establish a bijection

$$
f: B(J, K) \longrightarrow \bigcup_{\ell}(B(J, \ell) \times B(\ell, K)),
$$

where the union is disjoint. The bijection $f$ is very easy to define: A basis web of $w \in W(J K)$ has a minimal cut path, where a cut path is a path whose endpoints separate $J$ from $K$. A cut path is minimal if it crosses as few strands as possible:

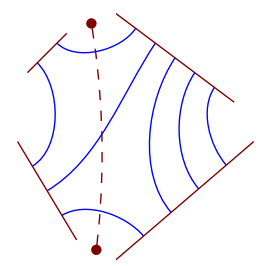

Let $w_{1}$ and $w_{2}$ be the two resulting webs. The cut path crosses some $i$ strands, and since it is minimal, there can be no U-turns among the $i$ strands in either $w_{1}$ or $w_{2}$. Thus, the relation $f(w)=\left(w_{1}, w_{2}\right)$ defines the desired map $f$. It is routine to check that $f$ is both injective and surjective.

Note that minimal cut paths are also useful in practice for generating the basis of a clasped web space.

Exercise 2.5. List the elements of $B(2,2,2,2,2)$.

\section{Precise Definition of A SPIDER}

In this section, we give a precise definition of a spider. The main way in which the general notion of a spider is more complicated than the example is that strands may be oriented and there may be more than one type of strand. Indeed, even in the $A_{1}$ case, we may consider $n$ parallel strands as equivalent to one strand labelled with $n$, as is already suggested by the notation.

A spider has a strand set $S$ which is a unital semigroup with unit $\emptyset$. In most of the examples in the paper, $S$ is a free, non-abelian semigroup. The strand set has an anti-involution $*: S \rightarrow S$ called duality or orientation reversal. For each $s \in S$, there is a web space $W(s)$, which may be just a set. For each $a$, there is a distinguished web $\beta_{a} \in W\left(a a^{*}\right)$, called a bare strand, and $1=\beta_{\emptyset}$ is called the empty web. Finally, there are three operations that exist for every $a$ and $b$ :

1. Join $\aleph_{a, b}: W(a) \times W(b) \rightarrow W(a b)$.

2. Rotation $\rho_{a, b}: W(a b) \rightarrow W(b a)$.

3. Stitch $\sigma_{a, b}: W\left(a a^{*} b\right) \rightarrow W(b)$. 
The subscripts of the operations may be dropped when they are clear from context. A spider may in addition be defined in a (symmetric tensor) category other than the category of sets. For example, an additive spider is one in which web spaces are abelian groups, rotation and stitch are additive, and join is additive, while in a linear spider, the web spaces are vector spaces and the operations are linear or bilinear.

The three spider operations must satisfy the following axioms, which are divided into groups according to which operations they involve. We define $\sigma_{b, a, c}=\rho_{c, b} \sigma_{a, c b} \rho_{b, a a^{*} c}$ for brevity in the last three axioms.

- Rotation only:

1. $\rho_{a, \emptyset}=\rho_{\emptyset, a}=\mathrm{id}$.

2. $\rho_{a, b c} \rho_{c, a b} \rho_{b, c a}=\mathrm{id}$.

3. $\rho_{a, a^{*}}\left(\beta_{a}\right)=\beta_{a^{*}}$

- Join only:

4. $(u \bowtie v) \bowtie w)=u \bowtie(v \bowtie w)$

5. $u \bowtie 1=u$

- Join and rotation:

6. $\rho_{a, b}\left(u \bowtie_{a b, \emptyset} v\right)=\rho_{a, b}(u) \aleph_{b a, \emptyset} v$

7. $\beta_{a b}=\rho_{a^{*}, a b b^{*}}\left(\beta_{a^{*}} \bowtie \beta_{b}\right)$

8. $\rho_{a, b}\left(u \bowtie_{a, b} v\right)=v \aleph_{b, a} u$

- Rotation and stitch:

9. $\sigma_{a, b d} \sigma_{a a^{*} b, c, d}=\sigma_{b, c, d} \sigma_{a, b c c^{*} d}$

10. $\sigma_{a, \emptyset}=\sigma_{a^{*}, \emptyset} \rho_{a, a^{*}}$.

- Join, rotation, and stitch:

11. $\sigma_{a, a^{*}, b}\left(\beta_{a} \aleph_{a a^{*}, a b} u\right)=u$

12. $\sigma_{b, a, c}\left(u \bowtie_{b a, a^{*} c} v\right)=\rho_{b, c}\left(\rho_{a^{*}, c}(v) \bowtie_{c a^{*}, a b} \rho_{b, a}(u)\right)$.

The formal spider axioms are embarrassingly complicated, but they can be phrased in more natural (if less formal) terms. In particular, the following compound web principle is equivalent to axioms 1-7 and 10-12. To state the principle, we first define a pre-spider to be an algebraic object satisfying these 10 axioms, but not necessarily axioms 8 and 9 .

Let $U$ be the free semigroup generated by a set $X$, and let $*$ be any involution of $X$ extended to an anti-involution of $U$. Let $L_{u}$ be an abstract set of labels for each $u \in U$ and let $L$ be the disjoint union. Consider a graph $G$ in a disk such that the vertices of $G$ on the boundary are univalent, such that each edge is labelled by an element of $x \in X$ and oriented unless $x=x^{*}$, and such that one of the vertices at the boundary is distinguished as first, and such that one edge of each internal vertex is distinguished as first. The edges incident to an internal vertex $v$ are then linearly ordered going counterclockwise around $v$; let $x_{i}$ be the label of the $i$ th edge $e$ of $v$ if the edge is unoriented or oriented outward, and let $x_{i}$ be the dual of the label of $e$ if it is oriented inward. Each $v$ should be labelled by an element of $L_{x_{1} \ldots x_{n}}$. Then we define the web space $W\left(x_{1} \ldots x_{n}\right)$ as the set of all graphs $G$ with boundary labelled $x_{1}, \ldots, x_{n}$ (following the same convention of taking the dual when an edge at the boundary is oriented inward), considered up to isotopy and up to the modification of reversing an edge and dualizing its label.

We define the free pre-spider $\mathcal{S}(X, L)$ by defining the spider operations in the same way as for the combinatorial $A_{1}$ spider: Join is given by band-connected sum, rotation is given by changing which boundary point is first, and stitch is given by connecting two adjacent boundary points by an arc. A tedious but straightforward computation demonstrates that $\mathcal{S}(X, L)$ satisfies axioms 1-7 and 10-12. Conversely, suppose that an algebraic object $\mathcal{S}$ consists of a strand set $S$ and a collection of web spaces $\{W(s)\}$ with operations of join, rotation, and stitch. Then the compound web principle stipulates that any $*$-preserving map $f: X \rightarrow S$ and any set of maps $\left\{L_{u} \rightarrow W(f(u))\right\}$ extend to a morphism $\mathcal{S}(X, L) \rightarrow \mathcal{S}$ preserving join, rotation, and stitch. The compound web principle is equivalent to the statement that $\mathcal{S}$ is a pre-spider. Informally, in a pre-spider $\mathcal{S}$, if a sequence of joins, rotations, and stitches are denoted by a planar diagram whose connecting arcs are the stitches, the resulting web depends only on the diagram and not on the order of the individual operations. The remaining two axioms for $\mathcal{S}$ can be understood as follows: Axiom 8 says that if a disconnected component of a compound web is moved from one face of the remaining part of the web to another, it does not change the value of the web. Axiom 9 says that the value of a boundaryless compound web depends only on its embedding in the sphere and not on its embedding in the disk.

Although the above definitions are not completely standard, a spider can also be defined in categorytheoretic terms. A spider is a (small) strict monoidal category, which is a category with an associative but 
not necessarily commutative tensor product $\otimes$. Moreover, a spider must be pivotal (or rigid), which means that there is a canonical isomorphism $V^{* *} \cong V$, and spherical, a condition which is equivalent to axiom 9 . See Barrett and Westbury [1] for a careful exposition of spherical categories. Given any spherical category, the strand set of the corresponding spider is precisely the set of objects of the category, and its web space $W(V)$ is defined as $\operatorname{Inv}(V)=\operatorname{Hom}(T, V)$, where $T$ is the trivial object, which serves as the identity of the tensor product operation. The spider operations are then defined in terms of monoidal category operations in the same way as for the algebraic $A_{1}$ spider.

We review the spiders defined so far in terms of these definitions. The strand set of the combinatorial and algebraic $A_{1}$ spiders is a free semigroup with one generator; for $n \in \mathbb{Z}_{\geq 0}$, a strand with strand type $n$ is synonymous with $n$ parallel strands. The clasped combinatorial $A_{1}$ spider is a different object and its strand set is a free semigroup with countably many generators, namely the different clasps.

It is useful to treat an additive or linear spider or pre-spider as a ring, and to consider the usual constructions with rings such as morphisms, ideals, and quotients. An ideal $\mathcal{I}$ in a linear pre-spider $\mathcal{S}$ is a collection of linear subspaces $I(s) \subset W(s)$ which are closed under rotation and stitch and closed under join with an arbitrary web in $\mathcal{S}$. Clearly, if $\mathcal{I}$ is an ideal, the quotient spaces $W(s) / I(s)$ form a pre-spider $\mathcal{S} / \mathcal{I}$. Given $X$ and $L$ as above, we can form the free linear pre-spider $\overline{\mathcal{S}}(X, L)$ as the linear extension of $\mathcal{S}(X, L)$. Many spiders (albeit only those whose strand set is a free semigroup) can be defined in terms of generators and relations, meaning that such a spider is a quotient of $\overline{\mathcal{S}}(X, L)$ by the ideal generated by an arbitrary set of relators. Indeed, the combinatorial $A_{1}$ spider is defined in exactly this fashion.

Note that in an additive pre-spider, the web space $W(\emptyset)$ is a commutative ring, and the other web spaces become $W(\emptyset)$-modules under join. Axiom 8 guarantees that rotation and stitch are module endomorphisms, so that an additive spider is automatically a $W(\emptyset)$-module spider, and in particular it is a linear spider if $W(\emptyset)$ is a field. See also Barrett and Westbury [1].

The category of finite-dimensional representations of any quantum Lie group $U_{q}(\mathfrak{g})$ with $\mathfrak{g}$ a complex simple Lie algebra is spherical, and therefore yields a spider. Technically, this is not a small category, meaning that the collection of all objects is too large to be a set, but we can obtain an equivalent small category by taking a single representative of each isomorphism class of finite-dimensional representations.

In particular, self-dual representations correspond to self-dual or unoriented strands, but therein lies a technicality and a potential sign error. An unoriented strand in a spider can only correspond to a symmetrically self-dual representation, while many representations (for example the representation $V_{n}$ of sl(2) for $n$ odd) are antisymmetrically self-dual. If there is to only one strand for each isomorphism class, each self-dual representation must be defined as a $\mathbb{Z} / 2$-graded vector space in which the antisymmetric part has an odd grading. Then any self-dual representation of any $\mathfrak{g}$ has a graded-symmetric invariant bilinear form, and the representation can correspond to an unoriented strand. This is why rotation in the combinatorial $A_{1}$ spider differs by a sign from ordinary cyclic permutation of tensor factors in $\operatorname{Inv}\left(V_{1}^{\otimes n}\right)$; it exactly equals graded cyclic permutation. Among representations of rank two Lie algebras, the representation $V\left(a \lambda_{1}+b \lambda_{2}\right)$ of $B_{2}$ also has an odd grading when $a$ is odd.

Given a Lie algebra $\mathfrak{g}$, the subcategory of irreducible representations and their tensor products also yields a spider, which we will call the clasped algebraic $\mathfrak{g}$ spider. The unclasped algebraic $\mathfrak{g}$ spider is the subcategory whose objects are the fundamental irreducible representations (those whose heighest weight is a simple weight) and their tensor products. In the rest of the paper, we will define combinatorial $\mathfrak{g}$ spiders when $\mathfrak{g}$ has rank 2, and we will show that they are isomorphic to their algebraic counterparts.

\section{THE COMBINATORIAL RANK 2 SPIDERS}

For convenience, let $\mathbb{C}$ with $q \in \mathbb{C}$ or $\mathbb{C}\left(q, q^{1 / 2}, q^{1 / 3}, \ldots\right)$ be the ground field. The unclasped combinatorial rank 2 spiders describe the invariants of tensor products of the two fundamental representations $V\left(\lambda_{1}\right)$ and $V\left(\lambda_{2}\right)$ of each of the Lie algebras $A_{2}, B_{2}$, and $G_{2}$. These two representations are duals of each other for $A_{2}$ and are self-dual in the other two cases. The strand set for the combinatorial $A_{2}$ spider is defined as the free semigroup of strings of symbols "+" and "-", which correspond to $V\left(\lambda_{1}\right)$ and $V\left(\lambda_{2}\right)$, respectively. The dual of a sign string is given by reversing the string and flipping the signs; for example,

$$
(+--++)^{*}=--++- \text {. }
$$

In the $B_{2}$ and $G_{2}$ spiders, the strand set is the free semigroup of strings of self-dual symbols "1" and " 2 ", so that duality is just string reversal. 
Given a sign string $s=s_{1} \ldots s_{n}$, define the $A_{2}$ basis web set $B(s)$ to be the set of non-elliptic, bipartite, trivalent graphs properly embedded in a disk with boundary points labelled $s_{1}, \ldots, s_{n}$ in counter-clockwise order. By a trivalent graph properly embedded in a disk, we mean a 1-dimensional subset of the disk locally modelled by the following five allowed neighborhoods of a point in the disk:
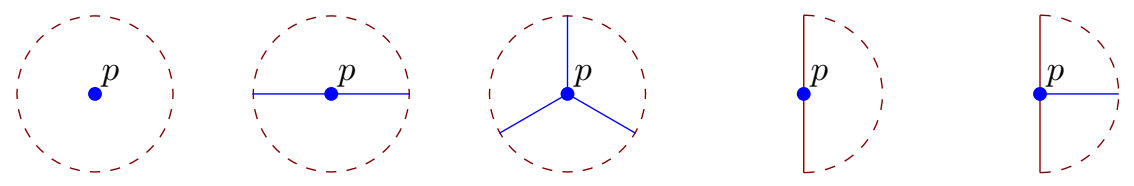

The allowed neighborhoods might be called empty disk, strand, trivalent vertex, empty boundary, and endpoint. Such a graph is bipartite if its endpoints are signed and its edges are oriented in such a way that the in-degree at each vertex is either 0 or 3, and such that edges point towards positive vertices and away from negative ones. Finally, such a graph is non-elliptic if all internal faces have at least six sides, where an internal face is a component of the complement of the graph that does not touch the boundary of the disk. These graphs, henceforth called basis webs, are considered up to isotopy relative to the boundary. For example, the 6 elements of $B(+++---)$ are
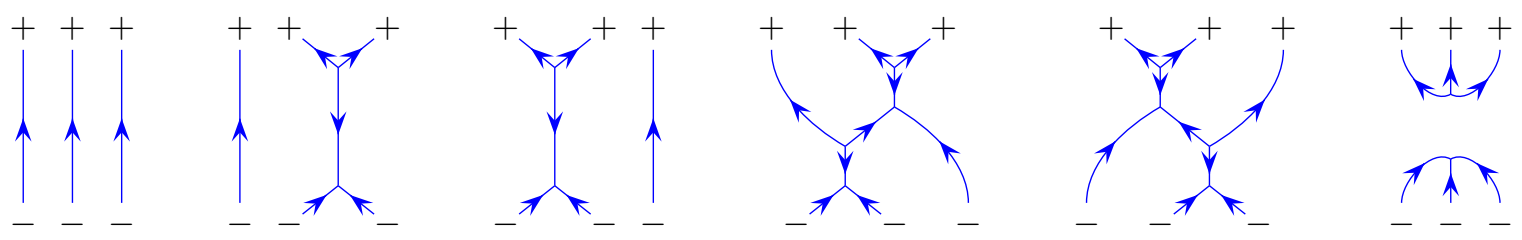

while

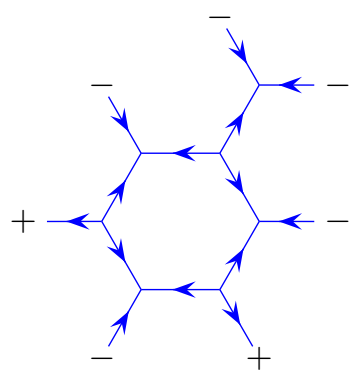

is an element of $B(+-+----)$.

The vector space of formal linear combinations of elements of $B\left(s_{1} \ldots s_{n}\right)$ is the $A_{2}$ web space $W\left(s_{1} \ldots s_{n}\right)$. Partly elliptic, bipartite, trivalent graphs in a disk, will denote webs also, although not basis webs. Specifically, each type of elliptic face is defined as a linear combination of basis webs according to the rules

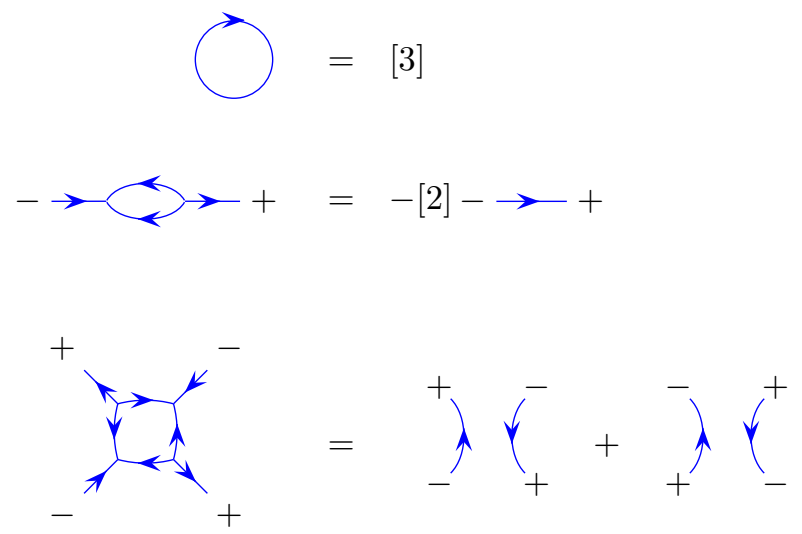


The value of a larger graph which contains an elliptic face is inductively defined by the same rules. For example,
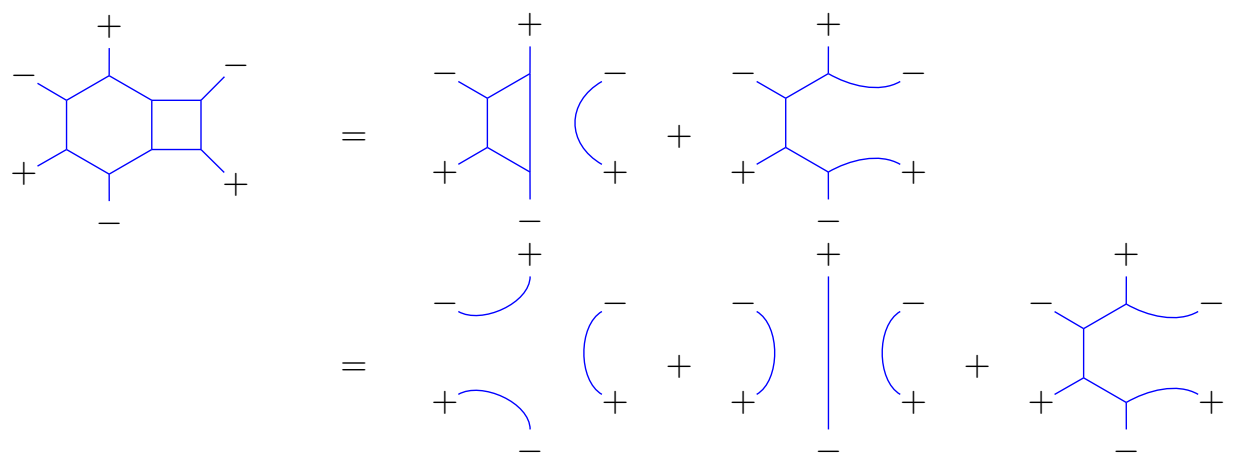

(Here and below, we may omit orientations of edges when they are clear from context.) Rotation, join, and stitch operations are defined in the usual graphical way: For basis webs, rotation is rotation of the disk, join is band-connected sum, and stitch is the operation of connecting two adjacent boundary points by an arc. Stitch is only defined when the two adjacent boundary points have opposite sign, to preserve the orientation structure of $A_{2}$ webs, and it may produce elliptic faces, which must be reduced to obtain a linear combination of basis webs. Compound webs, and in particular concatenation, are also defined either by extension of the three basic operations, or directly by the principle of reduction of elliptic faces.

Another way to phrase the definition of the $A_{2}$ spider is that it is generated by the two webs
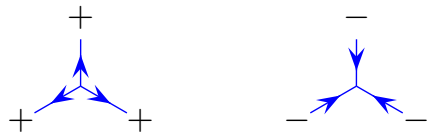

with equations (2) as relations. Similarly, the $A_{1}$ spider is trivially generated with the sole relation that a closed loop yields a factor of $a$.

The $B_{2}$ and $G_{2}$ spiders are also most conveniently defined by generators and relations. The $B_{2}$ spider is generated by the single web

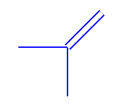

with the relations

$$
\begin{aligned}
& \bigcirc=-\left(q^{2}+q+q^{-1}+q^{-2}\right) \\
& \bigcirc=q^{3}+q+1+q^{-1}+q^{-3} \\
& =0 \\
& =\infty==-\left(q+2+q^{-1}\right)= \\
& C_{=0}=0 \\
& \aleph-\rangle\langle=\underbrace{-})
\end{aligned}
$$


where a strand is denoted by a double edge if its strand type is "2" and by a plain single edge if its strand type is " 1 ". The $G_{2}$ spider is generated by the webs

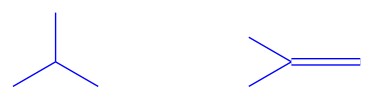

with the relations

$$
=q^{5}+q^{4}+q+1+q^{-1}+q^{-4}+q^{-5}
$$

To form a basis, we first note in the $G_{2}$ case that the last relation allows the elimination of all internal double edges, leaving only those with at least one vertex at the boundary. In the $B_{2}$ spider, we define a tetravalent vertex to achieve the same end:

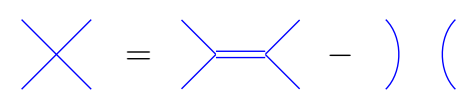


The tetravalent vertex then satisfies the relations

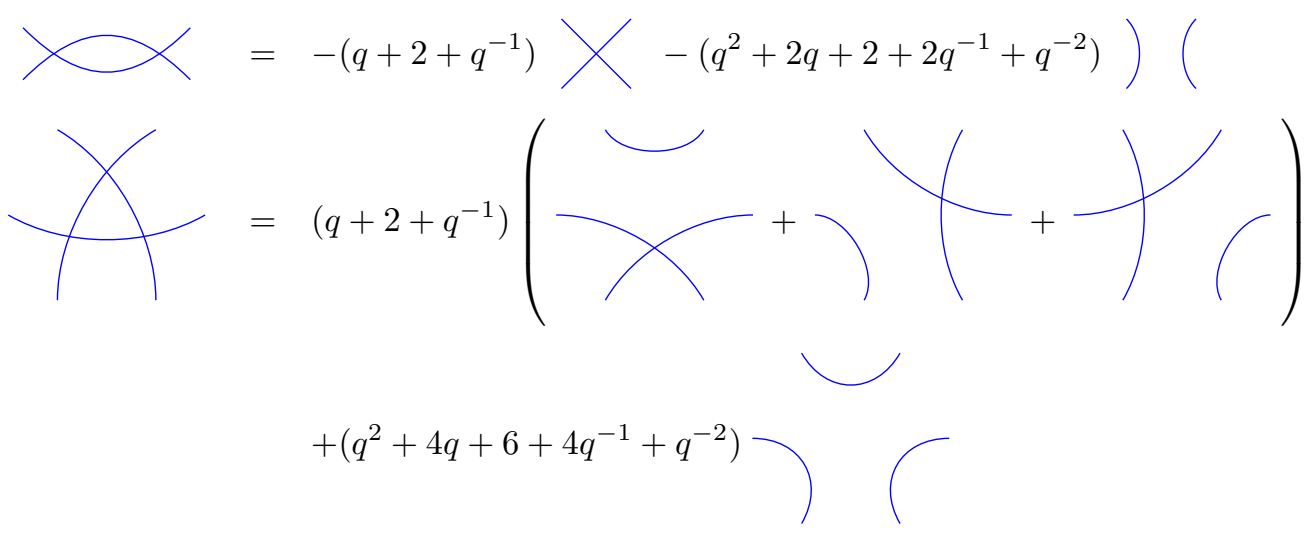

In both spiders, we can say that non-elliptic webs with no internal double edges are a basis, provided that we define formal angles of
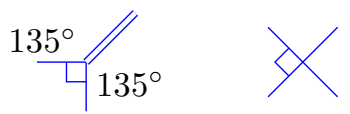

for the vertices in the $B_{2}$ spider and angles of

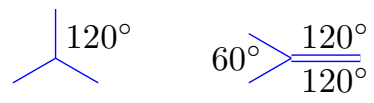

in the $G_{2}$ spider, and we declare that a face is elliptic if its total exterior angle is less than 360 degrees. For example, in the $G_{2}$ spider, the pentagon

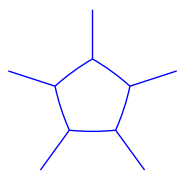

is elliptic, but the pentagon

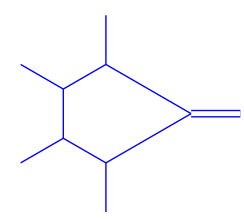

is flat rather than elliptic.

If we understand the rank two spiders in terms of generators and relations, then it is clear that nonelliptic webs linearly span all webs, but it is not obvious that there are linearly independent. Alternatively, if we understand the rank two spiders in terms of the non-elliptic bases, then it is not clear that the elliptic reduction equations are consistent. Put a third way, do two different reductions of a partly elliptic web always give the same linear combination of non-elliptic webs? The author [9], and independently Jaeger [4], established that the given coefficients are, up to trivial normalization, the only choices for which the equations are consistent. 
Rank 2 spiders also admit crossings that lead to link invariants, but the link invariants will not be discussed in this paper. There are two types of crossings in the $A_{2}$ spider:

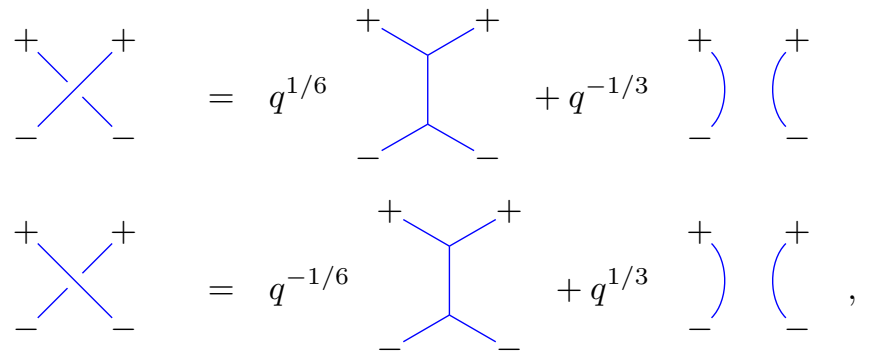

four types in the $B_{2}$ spider:

$$
\begin{aligned}
& \left.\searrow=-q^{1 / 2}\right)\left(-q^{-1 / 2} \leftrightharpoons+\frac{1}{q^{1 / 2}+q^{-1 / 2}}\right\rangle \\
& \left.\searrow=\frac{q^{-1 / 2}}{q^{1 / 2}+q^{-1 / 2}} \longrightarrow+\frac{q^{1 / 2}}{q^{-1 / 2}+q^{1 / 2}}\right\rangle \\
& \searrow=\frac{q^{1 / 2}}{q^{1 / 2}+q^{-1 / 2}} \longrightarrow+\frac{q^{-1 / 2}}{q^{-1 / 2}+q^{1 / 2}} \geqslant \\
& \Downarrow /(=q))\left(\left(+q^{-1} \Longleftarrow+\frac{1}{q+2+q^{-1}}\right.\right.
\end{aligned}
$$

and four types in the $G_{2}$ spider

$$
\begin{aligned}
& \left.\searrow=\frac{q^{-1 / 2}}{q^{1 / 2}+q^{-1 / 2}} \backslash+\frac{q^{1 / 2}}{q^{1 / 2}+q^{-1 / 2}}\right\rangle\left\langle+\frac{q^{-3 / 2}}{q^{1 / 2}+q^{-1 / 2}} \leftrightharpoons+\frac{q^{3 / 2}}{q^{1 / 2}+q^{-1 / 2}}\right)( \\
& \searrow=-\frac{q^{-3 / 2}}{q^{1 / 2}+q^{-1 / 2}} \searrow^{\prime}-\frac{q^{3 / 2}}{q^{1 / 2}+q^{-1 / 2}} \geqslant<-\frac{1}{q+2+q^{-1}} \\
& \left.\searrow=-\frac{q^{3 / 2}}{q^{1 / 2}+q^{-1 / 2}} \searrow-\frac{q^{-3 / 2}}{q^{1 / 2}+q^{-1 / 2}} \geqslant-\frac{1}{q+2+q^{-1}}\right) \\
& \left.\left.\Downarrow=\frac{q^{5}-q^{3}+q-q^{-3}}{q^{2}-1+q^{-2}}\right)\right)\left(\left(+\frac{-q^{3}+q-q^{-3}-q^{-5}}{q^{2}-1+q^{-2}} \Longleftarrow+\frac{1}{q+2+q^{-1}}\right.\right.
\end{aligned}
$$

Note that crossings are the first webs whose coefficients are not symmetric in $q$ and $q^{-1}$.

4.1. Clasps and clasped web spaces. The rank 2 spiders also admit clasps and clasped web spaces. As before, the type of a clasp is the same as an unclasped strand type, but in the rank 2 cases there are many more combinations. Our notation will be that if $s$ an unclasped strand type, $c=[s]$ is the corresponding clasp. We will also consider sequences of clasps $C=c_{1} c_{2} \ldots c_{n}=\left[s_{1}\right]\left[s_{2}\right] \ldots\left[s_{n}\right]$.

Clasps and clasped web spaces in the $A_{2}$ spider are the easiest to describe: Define the weight wt $(s)$ of a sign string $s n \lambda_{1}+k \lambda_{2}$ if there are $n$ plusses and $k$ minuses. Recall the usual partial ordering of the weight 
lattice of lattice of $A_{2}$ : it is generated by

$$
\begin{aligned}
& a \lambda_{1}+b \lambda_{2} \succ(a+1) \lambda_{1}+(b-2) \lambda_{2} \\
& a \lambda_{1}+b \lambda_{2} \succ(a-2) \lambda_{1}+(b+1) \lambda_{2} .
\end{aligned}
$$

There is a clasped web space $W(C)$ for each possible clasp sequence $C$. For example, $W([+-+][-+-][-+])$ denotes a clasped web space. The basis $B\left(\left[s_{1}\right]\left[s_{2}\right] \ldots\left[s_{k}\right]\right)$ of $W\left(\left[s_{1}\right]\left[s_{2}\right] \ldots\left[s_{k}\right]\right)$ is a subset of the set of unclasped basis webs $B\left(s_{1} s_{2} \ldots s_{k}\right)$ consisting of those non-elliptic webs with non-convex clasps. Here an (external) clasp is non-convex if it has the property that the weight of any path between endpoints of the clasp that is transverse to the web (a cut path) is greater than or equal to the weight of the clasp; the weight of such a path is defined as the number of strands that cross it in the direction of the clasp and the number that cross away from the clasp. For example, the following web has a partly convex clasp, because its weight is $2 \lambda_{1}+\lambda_{2}$, but there is an arc with weight $2 \lambda_{2}$ that cuts off the clasp:

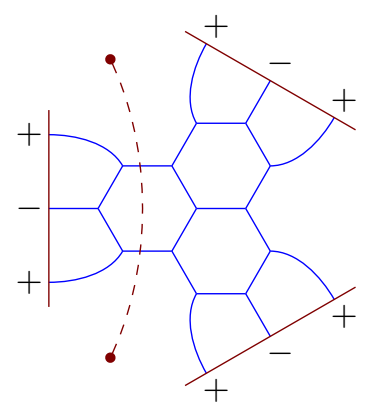

It is therefore not a basis web of $W([+-+][+-+][+-+])$. Clasped web spaces can again be interpreted as both quotients and subspaces of unclasped web spaces. Any web with at least one partly convex clasp is understood as the zero vector. By this convention and equations (2), any trivalent graph in a disk with suitable boundary can be interpreted as some vector in the clasped web space.

The non-convexity condition for clasps may seems unnecessarily strong. One might alternatively stipulate that every cut path cross at least as many strands as the number of strands in the clasp, or that the weight of no cut path be strictly less than the weight of the clasp. However, by Lemma 6.5, the two condictions are both equivalent to the one given. Say that a cut path of a web is minimal if its weight is minimal with respect to the partial ordering. Then in particular, Lemma 6.5 implies that all minimal cut paths with a fixed pair of endpoints have the same weight, the minimal cut weight.

As usual, join and rotation in the clasped $A_{2}$ spider are straightforward, but stitch involves internal clasps. An internal clasp of type $s$ is defined as an idempotent in the unclasped web space $W\left(s^{*} s\right)$ that annihilates any web in $W\left(s^{*} t\right)$ with $\operatorname{wt}(t) \prec \operatorname{wt}(s)$. For example,

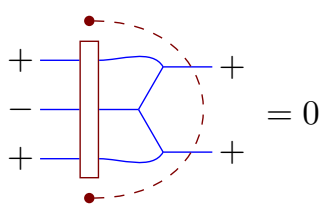

It is not clear that internal clasps exist; without them, we must understand the clasped spider not as a spider but as a collection of web spaces with the operations of rotation and join only. We will use this incomplete structure in an indirect argument that internal clasps must exist for all three rank 2 cases (as before, they are highest-weight projections), but in the $A_{2}$ case we will also give a more explicit construction.

The construction of the clasped $B_{2}$ spider is entirely analagous to that of the clasped $A_{2}$ spider, except that the definition of a cut path and its weight are slightly different. A cut path may cut diagonally through a tetravalent vertex, and its weight is defined as $n \lambda_{1}+\left(k+k^{\prime}\right) \lambda_{2}$, where $n$ is the number of type " 1 " strands that it cuts, $k$ is the number of type " 2 " strands that it cuts, and $k^{\prime}$ is the number of tetravalent vertices 
that it bisects. For example, the following cut path has weight $\lambda_{1}+2 \lambda_{2}$ :

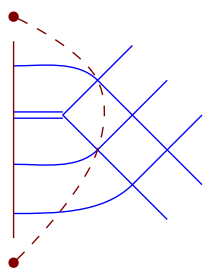

Recall that there is a natural partial ordering of the $B_{2}$ weight lattice given by

$$
\begin{aligned}
& a \lambda_{1}+b \lambda_{2} \succ(a-2) \lambda_{1}+(b+1) \lambda_{2} \\
& a \lambda_{1}+b \lambda_{2} \succ(a+2) \lambda_{1}+(b-2) \lambda_{2} .
\end{aligned}
$$

The clasped $G_{2}$ spider has a more significant difference. A cut path may contain a type " 1 " strand in its interior, and its weight is $n \lambda_{1}+\left(k+k^{\prime}\right) \lambda_{2}$, where $n$ is the number of type " 1 " strands that it cuts, $k$ is the number of type " 2 " strands that it cuts, and $k$ ' is the number of type " 1 " strands that it contains. For example, the following cut path has weight $\lambda_{1}+2 \lambda_{2}$ :

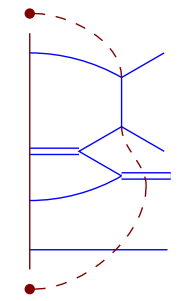

As before, the webs with non-convex clasps, where non-convexity is defined using the partial ordering

$$
\begin{aligned}
& a \lambda_{1}+b \lambda_{2} \succ(a-2) \lambda_{1}+(b+1) \lambda_{2} \\
& a \lambda_{1}+b \lambda_{2} \succ(a+3) \lambda_{1}+(b-2) \lambda_{2}
\end{aligned}
$$

in the $G_{2}$ weight lattice, form a basis of each clasped web space. The more significant difference is that a basis element of the unclasped web space with a convex clasp is not necessarily zero. Rather, if a web, whether non-elliptic or not, has a cut path which cuts of a clasp, which does not contain any type " 1 " strands and whose weight is less than that of the clasp, then the web is zero. For example,

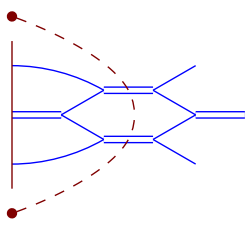

It may not be immediate that the kernel of this quotienting operation complements the subspace defined as the clasped web space. This will be shown in Section 6.2.

This concludes the definition of the combinatorial rank 2 spiders and the definition of clasped web spaces. Only the operation of stitch, which depends on the existence of internal clasps, remains to be fully defined.

\section{The MORPhism FROM COMBINATORIAL TO ALGEBRAiC}

Let $V_{+}$and $V_{-}$be the two fundamental representations of $A_{2} \cong \operatorname{sl}(3)$, with $V_{-} \cong\left(V_{+}\right)^{*}$. Let $V_{1}$ and $V_{2}$ be the two fundamental representations of $B_{2} \cong \operatorname{sp}(4) \cong \operatorname{so}(5)$, and give the two fundamental representations of $G_{2}$ the same names with $\operatorname{dim} V_{1}<\operatorname{dim} V_{2}$ in both cases. Then the vector spaces

$$
\begin{aligned}
& \operatorname{Inv}_{A_{2}}\left(V_{+}^{\otimes 3}\right) \\
& \operatorname{Inv}_{A_{2}}\left(V_{-}^{\otimes 3}\right) \\
& \operatorname{Inv}_{B_{2}}\left(V_{1} \otimes V_{1} \otimes V_{2}\right) \\
& \operatorname{Inv}_{G_{2}}\left(V_{1}^{\otimes 3}\right) \\
& \operatorname{Inv}_{G_{2}}\left(V_{1} \otimes V_{1} \otimes V_{2}\right)
\end{aligned}
$$


are all 1-dimensional. When $q=1$, and for each of $A_{2}, B_{2}$, and $G_{2}$, there exists a morphism $\Phi$ from the unclasped combinatorial spider to the unclasped algebraic spider with the following property. If $s$ a generator of the strand set, then $\Phi(s)=V_{s}$, and if $T$ is a trivalent vertex of some type, then $\Phi(T)$ is a non-zero element in one of the above invariant spaces. We sketch the argument for the existence of $\Phi$ in the $A_{2}$ case (see Reference 9 for details): Pick any two non-zero elements $x \in \operatorname{Inv}_{A_{2}}\left(V_{+}^{\otimes 3}\right)$ and $x^{*} \in \operatorname{Inv}_{A_{2}}\left(V_{-}^{\otimes 3}\right)$. By counting dimensions of invariant spaces, each of the left sides of equations (2), (3), and (何) must be some linear combinations of the right sides in the algebraic $A_{2}$ spider, if $x$ and $x^{*}$ are denoted by the usual trivalent vertices. But at the same time, a computation shows that the right sides are linearly independent in the algebraic $A_{2}$ spider, and that, up to normalization of $x$ and $x^{*}$, the given coefficients are the only ones that respect this normalization. Thus, after rescaling, the invariant tensors $x$ and $x^{*}$ of $U_{q}\left(A_{2}\right)$ must satisfy the relations of the combinatorial $A_{2}$ spider, although perhaps with a different choice of $q$. Another simple computation shows that the choice of $q$ is the same. Thus we can set $\Phi(T)=x$ and $\Phi\left(T^{*}\right)=x^{*}$, if $T$ and $T^{*}$ are the trivalent vertices that generate $A_{2}$.

Theorem 5.1. The morphism $\Phi$ from the combinatorial to the algebraic $A_{2}, B_{2}$, or $G_{2}$ spider is surjective when $q=1$, and therefore for generic $q$.

This theorem is proved in Reference 9, but we give a more conceptual argument here:

Proof: Let $q=1$, let $\mathfrak{g}$ be $A_{2}, B_{2}$, or $G_{2}$, and let $G$ be the compact, simply-connected Lie group whose complexified Lie algebra is $\mathfrak{g}$. If $s=s_{1} \ldots s_{n}$ is a string, define

$$
V_{s}=V_{s_{1}} \otimes \ldots V_{s_{n}} \text {. }
$$

In each case, the image $\mathcal{X}$ of $\Phi$ is some subspider of the algebraic spider of $\mathfrak{g}$. We interpret $\mathcal{X}$ as a category of linear transformations between tensor products of fundamental representations. The category $\mathcal{X}$ contains switching maps $x \otimes y \mapsto y \otimes x$, since they are the images of crossings under the map $\Phi$. Moreover, each End $\mathcal{X}\left(V_{s}\right)$ is a semisimple algebra, by the following construction: We define the Hermitian adjoint $w^{*}$ of $w \in W\left(s s^{*}\right)$ by reflecting $w$ about a vertical axis, taking the complex conjugate of all coefficients, and, in the $A_{2}$ spider, reversing all orientations and signs:

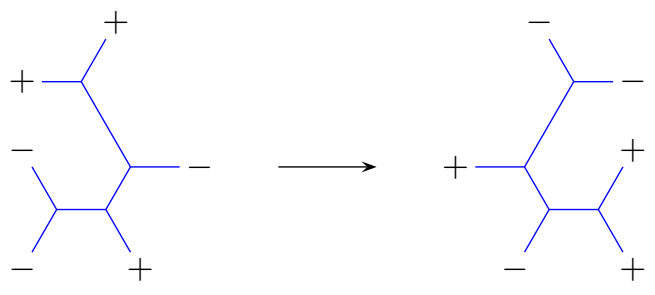

The morphism $\Phi$ intertwines the combinatorial Hermitian adjoint with the usual Hermitian adjoint in the algebraic spider. Since $\operatorname{End}_{\mathcal{X}}\left(V_{s}\right)$ is closed under Hermitian adjoint, it must be semisimple.

The category $\mathcal{X}$ does not contain the kernels and co-kernels of its morphisms, but it may be completed to a bigger category $\mathcal{X}^{\prime}$, in which $\mathcal{X}$ is a full subcategory, by adding these vector spaces as new objects. The category $\mathcal{X}^{\prime}$ then satisfies the hypotheses of the Tannaka-Krein duality theorem [8, p. 177], and must be the category of finite-dimensional representations of some compact group $H$. On the one hand, $H \subseteq G$, since everything in $\mathcal{X}^{\prime}$ is invariant under $G$. On the other hand, $H$ cannot be any bigger than $G$, because for each choice of $\mathfrak{g}, G$ is a maximal compact subgroup of the symmetry group of $\Phi(t)$ for a vertex $t$. (For example, the symmetry group of a non-zero element of $\operatorname{Inv}_{B_{2}}\left(V_{1} \otimes V_{1} \otimes V_{2}\right)$ is $\operatorname{sp}(4, \mathbb{C})$, with maximal compact subgroup $\operatorname{Spin}(5)$.) Therefore $\mathcal{X}^{\prime}$ is equivalent to the representation category of $U(\mathfrak{g})$, and $\mathcal{X}$ coincides with the algebraic $\mathfrak{g}$ spider.

It is more difficult to show that $\Phi$ is injective. We will prove this in Section 6 by demonstrating that the clasped web spaces and the web spaces of the clasped algebraic spider have equinumerous bases. If this is so for clasped web spaces, then it is also true for unclasped web spaces, which demonstrates that $\Phi$ is an isomorphism between unclasped spiders. We can then define an internal clasp as $\Phi^{-1}(\pi)$, where $\pi$ is the highest-weight projection from any strand in the algebraic $\mathfrak{g}$ spider to itself. This completes the definition of the clasped spiders, provided we verify the following lemma to show that $\Phi$ maps stitch to contraction.

Lemma 5.2. Suppose that $s$ and $t$ are strand types and $w \in W($ st $)$ is zero in the clasped web space $W([s] t)$. Then $w$ is annihilated by an internal clasp of type $[s]$. 
Proof: We assume the injectivity of $\Phi$ for unclasped web spaces, and we assume that $w \in B(s t)$ is a basis web. Let $\lambda$ be the weight of $s$, and let $w^{\prime}$ be $w$ with an internal clasp attached The tensor $\Phi(w)$ may be interpreted as a homomorphism, in particular as a composotion $V_{s} \rightarrow V(\lambda) \rightarrow V_{u} \rightarrow V_{t}$, where $u$ is the transverse strand type of a minimal cut path that separates $s$ from $t$ in $w$. By hypothesis, the weight of $u$ is lower than that of $s$. Therefore any map $V(\lambda) \rightarrow V_{u}$ must vanish, so $w^{\prime}$ must vanish.

The following result is then a corollary of the discussion of this section:

Theorem 5.3. The morphism $\Phi$ from the combinatorial to algebraic rank two spiders extends to clasped spiders.

\section{Equinumeration}

\subsection{The $A_{2}$ case.}

Theorem 6.1. Let $C$ be an $A_{2}$ clasp sequence and let wt $C$ be the corresponding sequence of weights. Then the vector spaces $W(C)$ and $\operatorname{Inv}(V($ wt $C))$ have the same dimension.

One of the main steps of the proof of Theorem 6.1 is the same as that of the proof of Theorem 2.3: Given two clasp sequences $C$ and $D$, there is a decomposition of vector spaces

$$
\operatorname{Inv}(V(\text { wt } C) \otimes V(\text { wt } D)) \cong \bigoplus_{\mu} \operatorname{Inv}(V(\text { wt } S) \otimes V(\mu)) \otimes \operatorname{Inv}\left(V(\mu)^{*} \otimes V(\text { wt } D)\right) .
$$

We wish to prove the corresponding decomposition of sets of basis webs:

Theorem 6.2. Given two clasp sequences $C$ and $D$,

$$
B(C D) \cong \bigcup_{\lambda}\left(B\left(C c_{\lambda}\right) \times B\left(c_{\lambda}^{*} D\right)\right),
$$

where for each weight $\lambda, c_{\lambda}$ is some clasp with weight $\lambda$.

Theorem 6.2 is more complicated than its analogue for the $A_{1}$ spider, because there is no longer always a unique minimal cut path separating the clasp sequences $C$ and $D$ :

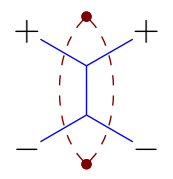

Although the two cut paths in this example have the same weight, they have different transverse strand types and they yield webs in different clasped web spaces. Yet not every ordering of the strands is always possible. Nevertheless, there is a way to reconcile these different decompositions and ameliorate the ordering problem.

Indeed, order independence is a large part of the combinatorial content of Theorem 6.1. For example, it implies the following result, which has an independent proof that is another warm-up to the proof of Theorem 6.1.

Theorem 6.3. If two sign strings $s_{1}$ and $s_{2}$ of the unclasped $A_{2}$ spider differ only in order, then $B\left(s_{1}\right)$ and $B\left(s_{2}\right)$ are equinumerous.

Proof: The web

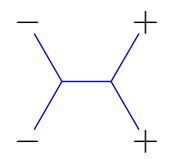

is the H-web. It suffices to consider the case in which $s_{1}$ and $s_{2}$ are the same except for one pair of transposed signs at adjacent positions $p$ and $q$. Then there is a bijection $h: B\left(s_{1}\right) \rightarrow B\left(s_{2}\right)$ that has the following effect 
on non-elliptic webs. If a web $w$ connects $p$ to $q$ by a bare strand, $h(w)$ is the same web with the orientation of the strand reversed:

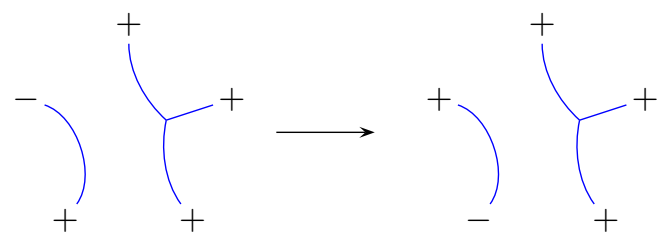

If there is an $\mathrm{H}$-web attached at $p$ and $q, h(w)$ is $w$ with the $\mathrm{H}$-web removed:

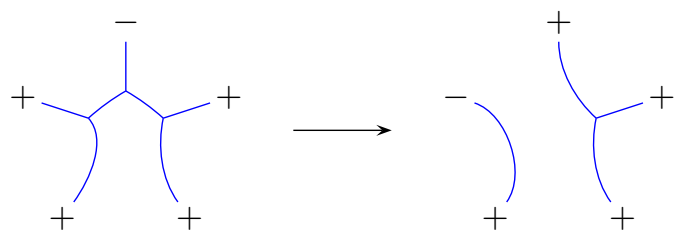

If there is no $\mathrm{H}$-web, then $h(w)$ is $w$ with an $\mathrm{H}$-web attached:

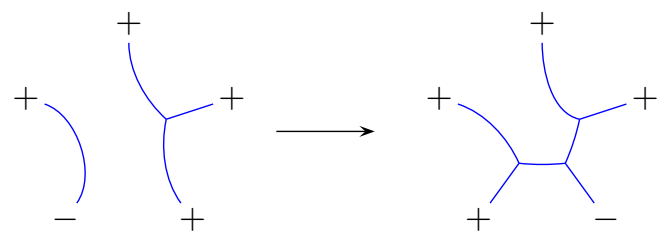

It is easy to check that $h(w)$ is non-elliptic if $w$ is, and that $h$ has an inverse. In fact, the inverse is also an $h$-map.

In the notation of the above proof, we also say that $w$ and $h(w)$ differ by an $\mathrm{H}$-move.

Lemma 6.4. If $\alpha$ is a minimal cut path of $w \in B(S T)$ separating $S$ from $T$, it divides $w$ into two parts $w_{1} \in B(S c)$ and $w_{2} \in B\left(c^{*} T\right)$ with non-convex clasps $c$ and $c^{*}$.

Proof: If $c$ were convex in $w_{1}, w_{1}$ would have a cut path $\alpha^{\prime}$ whose weight is lower than that of $c$, the same as the weight of $\alpha$. But $\alpha^{\prime}$ is also a cut path in $w$ and has the same endpoints as $\alpha$, contradicting the hypothesis that $\alpha$ is minimal:

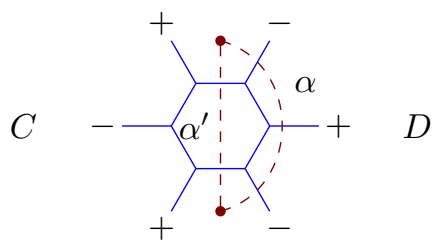

Lemma 6.5. If $\alpha$ and $\beta$ are cut paths from $p$ to $q$ of a basis web $w$ and $\alpha$ is minimal, then the weight of $\alpha$ is less than or equal to (and not incomparable to) the weight of $\beta$. If $\beta$ is also minimal, the two parts of $w$ cut by $\beta$ are the same as those of $w$ cut by $\alpha$ up to H-moves.

Proof: The proof is by induction on the complexity of $w$; assume that $w$ is a minimal counterexample. Assume also that, having chosen $w, \alpha$ and $\beta$ are transverse and intersect minimally. First, we can discard any structure of $w$ not bounded by $\alpha \cup \beta$; the new web has no clasps and is non-elliptic since $w$ is non-elliptic. Second, we claim that $\alpha$ and $\beta$ do not intersect except at their endpoints. For otherwise, let $\alpha^{\prime}$ be a segment of $\alpha$ between two consecutive intersections $x_{1}$ and $x_{2}$, and let $\beta^{\prime}$ be the arc of $\beta$ from $x_{1}$ to $x_{2}$ (which are not necessarily consecutive along $\beta$ ). Then the region between $\alpha^{\prime}$ and $\beta^{\prime}$ is either empty, in which case $\alpha^{\prime}$ and $\beta^{\prime}$ do not intersect minimally; or it constitutes a smaller counterexample than $w$ for some choice of $\alpha^{\prime}$; 
or $\beta^{\prime}$; or $\alpha$ and $\beta$ have comparable weight:

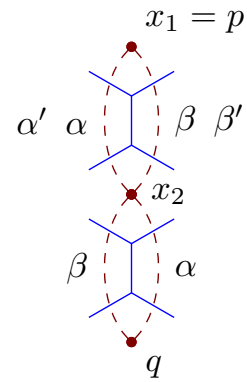

Third, $w$ must be connected, for if one of its connected components meets $\alpha$ but not $\beta, \alpha$ is not a minimal cut path; if one of its components meets $\beta$ but not $\alpha$, it may be discarded to produce a smaller counterexample; and if all components meet both $\alpha$ and $\beta$, one of them is a smaller counterexample:

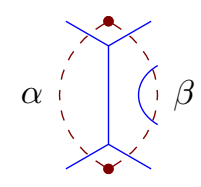

Finally, if $e_{1}$ and $e_{2}$ are adjacent endpoints of $w$, define the exterior curvature of the arc $\overline{e_{1} e_{2}}$ to be $180^{\circ}-n 60^{\circ}$, where $n$ is the number of vertices of $w$ connecting $e_{1}$ to $e_{2}$ :

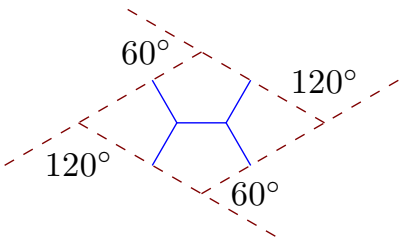

The total exterior curvature of $w$ is at least $360^{\circ}$ since $w$ is non-elliptic. Moreover, the curvature at the arcs containing $p$ and $q$ is at most $120^{\circ}$, for if it were $180^{\circ}$, either $w$ would be disconnected or it would be a single strand. Therefore there must be a segment $\gamma$ of either $\alpha$ or $\beta$ with positive curvature. There are three possibilities for the edges of $w$ that bound a face together with $\gamma$ :

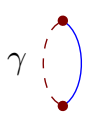

1

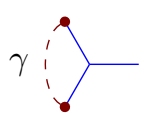

2

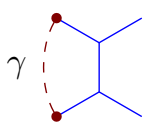

3

1. A "U". In this case, $w$ is either disconnected or a single strand.

2. A "Y". If $\gamma$ lies on $\alpha$, then $\alpha$ is not minimal. If $\gamma$ lies on $\beta$, then an isotopy of $\beta$ across the "Y" produces a smaller counterexample.

3. An " $\mathrm{H}$ ". In this case, an isotopy of either $\alpha$ or $\beta$ produces a smaller counterexample by an H-move.

This eliminates all possibilities for the least counterexample.

Lemma 6.6. Let $C$ be a sequence of clasps, let $c$ be an arbitrary clasp, and let $w \in B(C c)$ be a basis web. There is a cut path $\gamma$ that separates $c$ such that any other such cut path lies between $\gamma$ and $c$.

Proof: If $\alpha$ and $\beta$ are two transverse, minimal cut paths that separate $c$ and $p$ and $q$ are two consecutive transverse intersection points along either path, then the weight of the arc of $\alpha$ from $p$ to $q$ must equal the 
weight of the arc of $\beta$ from $p$ to $q$, for otherwise one path would provide a short-cut for the other and either $\alpha$ or $\beta$ would not be minimal. Thus the path $\gamma$ that follows the perimeter of $\alpha \cup \beta$ must also be minimal:

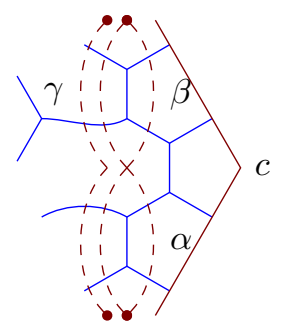

If we partially order cut paths by their distance from $c$, there is a unique maximal element.

Given $w$ as in Lemma 6.6, define the core of $w$ relative to $c$ to be the web $w^{\prime} \in B\left(C c^{\prime}\right)$ obtained by cutting away $c$ along the cut path $\gamma$ guaranteed by the lemma. Here $c^{\prime}$ is another clasp with the same weight as $c$. Let $B(C ; \lambda)$ be the set of all cores of webs $w \in B(C c)$ for all clasps $c$ of weight $\lambda$. Note that, since $c$ is non-convex in $w$, one of the minimal cut paths that separates $c$ is parallel to $c$. Therefore, by Lemma 6.5, $w$ differs from its core by H-moves. Note also that the core $w^{\prime}$ has the property that no $\mathrm{H}$-webs are attached at $c^{\prime}$, and that any web with this property is its own core.

We describe how an arbitrary basis web extends from one of its cores. Consider the following stair-step construction of a basis web from a core. Suppose that $w \in B(C[s])$ is a core relative to a clasp $[s]$ of weight $\lambda=a \lambda_{1}+b \lambda_{2}$, and suppose that $\left[s^{\prime}\right]$ is an arbitrary clasp of the same weight. Then the strings $s$ and $s^{\prime}$ each represent paths $p$ and $p^{\prime}$ from the the upper left corner to the lower right corner of an $a \times b$ rectangle, where each "+" in $s$ or $s^{\prime}$ is a step to the right and each "-" is a step down:

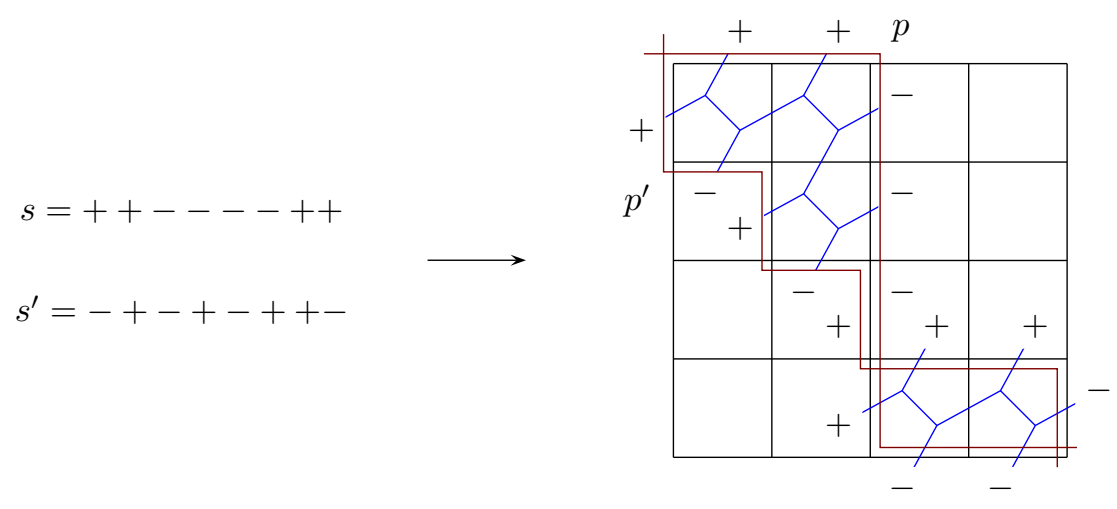

If an $\mathrm{H}$-web is placed in each square as indicated, the two paths $p$ and $p^{\prime}$ delineate a sequence of connected webs, separated by points or segments where $p$ and $p^{\prime}$ meet and may or may not cross. We attach each web bounded below by $p$ to $w$, and we invert and reverse the arrows of each web bounded above by $p$ and then attach it to $w$. The result is a web $w^{\prime} \in B\left(C\left[s^{\prime}\right]\right)$ with core $w$ :

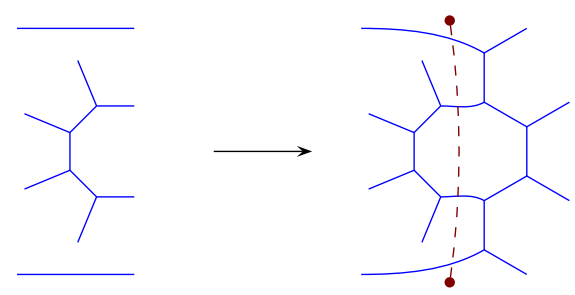

If $s_{1}$ and $s_{2}$ are arbitrary strings of the same weight, we can set $s^{\prime}$ to either in the stair-step construction, which implies that any core $w$ of a web in $B\left(C\left[s_{1}\right]\right)$ is also a core of a web in $B\left(C\left[s_{2}\right]\right)$. Moreover, it is easy to check that, if $w$ is fixed, the collection of webs so produced is closed under H-moves. Since every basis web is related to its core by H-moves, the stair-step construction produces all webs in $B(C c)$ for all $c$ with weight $\lambda$. In particular, we have demonstrated the following lemma: 
Lemma 6.7. Let $C$ be a sequence of clasps and let $c$ be a clasp of weight $\lambda$. If $w \in B(C[c])$, let $f(w)$ be its core. Then the map $f: B(C[c]) \rightarrow B(C ; \lambda)$ is a bijection.

Using the above lemmas, we can prove Theorem 6.2:

Proof: Let $w_{1} \in B(C ; \lambda)$ and $w_{2} \in B\left(D ; \lambda^{*}\right)$, and let $c_{1}$ and $c_{2}$ be the clasps of $w_{1}$ and $w_{2}$ of weight $\lambda$ and $\lambda^{*}$. Then we can either extend $w_{1}$ to a basis web in $B\left(C c_{2}^{*}\right)$ or extend $w_{2}$ to a basis web in $B\left(D c_{1}^{*}\right)$ and then sew the two webs together; by the symmetry of the stair-step construction, the resulting web $w \in B(C D)$ is the same in both cases. Define

$$
f: \bigcup_{\lambda}\left(B(C ; \lambda) \times B\left(D ; \lambda^{*}\right)\right) \rightarrow B(C D)
$$

by the above operation on pairs of cores. By Lemma 6.5 and the fact that H-moves preserve cores, there is also a map

$$
g: B(C D) \rightarrow \bigcup_{\lambda}\left(B(C ; \lambda) \times B\left(D ; \lambda^{*}\right)\right)
$$

given by splitting $w$ along a minimal cut path and taking the cores of the two halves. By Lemma 6.7, $f$ and $g$ are inverses. Using Lemma 6.7 again, we can see $g$ as the bijection claimed by the theorem.

To prove Theorem 6.1, we need one additional lemma. Say that a sign string $s$ is segregated if it is of the form

$$
++\ldots+--\ldots-
$$

a sign string with only +'s or only -'s is automatically segregated. Likewise, say that a clasp is segregated if it is of the form $[s]$ for a segregated sign string $s$.

Lemma 6.8. Let $c$ and $d$ be segregated clasps of weight $\lambda$ and $\mu$. Then the web basis set $B([+] c d)$ has has one element if and only if $\lambda^{*}=\mu+\lambda_{1} \mu^{*}=\lambda+\lambda_{1}$, or $\lambda^{*}=\mu+\lambda_{2}-\lambda_{1}$, and is empty otherwise.

Proof: Consider first three segregated clasps $c, d$, and $e$ (one of which might be empty) of arbitrary weight, and let $w \in B(c d e)$. We claim that $w$ must consist of a number of bare strands plus a flat component, as in the following example:

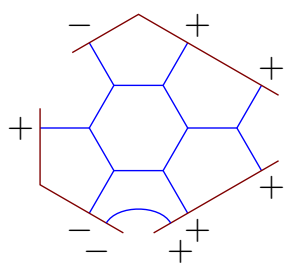

The argument is similar to the proof of Lemma 6.5. Assume first that $w$ is not connected. If $p$ and $q$ are adjacent endpoints of $w$, we define the curvature of the segment of the boundary of $w$ from $p$ to $q$ as in Lemma 6.5. Since $w$ is non-elliptic, it must one the one hand have total exterior curvature at most $360^{\circ}$. On the other hand, there can be no "U" or "Y" attached along $c$, $d$, or $e$, and in there is only one place along each clasp where an $H$ is possible. The curvature at this $H$, if it is present, is at most $60^{\circ}$, and the curvature elsewhere along the clasps is at most $0^{\circ}$. Moreover, unless $w$ is a bare strand, the curvature at the segments between the clasps is at most $60^{\circ}$ also. The largest possible total is $360^{\circ}$ exactly, so that $w$ is flat and its boundary is qualitatively a hexagon:

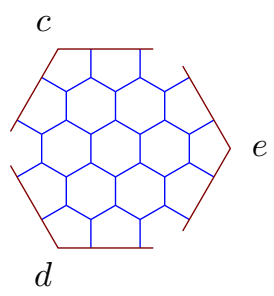

If $w$ is not connected, then the above argument applies to each connected component of $w$, with the additional observation that at most one component of $w$ can meet all three clasps $c, d$, and $e$. A component that only meets two clasps is necessarily a bare strand. 
In the case of interest, $e=[+]$. In this case, $w$ might consist entirely of bare strands, or it might have a component in the shape of a parallelogram or a trapezoid:
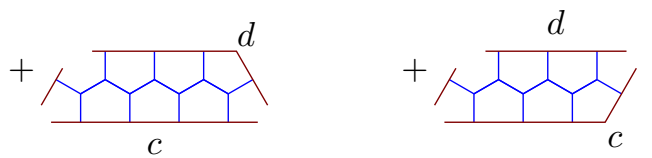

These possibilities exactly match the restrictions on the weights of $c$ and $d$.

Finally, we prove Theorem 6.1:

Proof: Let $\Lambda_{+}$be the set of all weights $\lambda=a \lambda_{1}+b \lambda_{2}$ with $a, b \in \mathbb{Z}_{\geq 0}$. Consider the abelian group $\mathbb{Z}\left[\Lambda_{+}\right]$of formal sums of elements $v(\lambda)$ for each $\lambda \in \Lambda_{+}$. Then we can define a product in $\mathbb{Z}\left[\Lambda_{+}\right]$using the dimensions of the clasped web spaces:

$$
v\left(\lambda_{1}\right) v\left(\lambda_{2}\right)=\sum_{\lambda_{3}}\left|B\left(c_{1} c_{2} c_{3}^{*}\right)\right| v\left(\lambda_{3}\right),
$$

where in the sum each $c_{i}$ is some clasp of weight $\lambda_{i}$. By Theorem 6.2, there exist bijections

$$
\bigcup_{d}\left(B\left(c_{1} c_{2} d\right) \times B\left(c_{3} c_{4} d^{*}\right)\right) \cong B\left(c_{1} c_{2} c_{3} c_{4}\right) \cong\left(\bigcup_{d} B\left(c_{2} c_{3} d\right) \times B\left(c_{4} c_{1} d^{*}\right)\right) .
$$

These bijections imply that multiplication is associative, and therefore $\mathbb{Z}\left[\Lambda_{+}\right]$is a ring. To establish Theorem 6.1, it suffices to check that the map $v(\lambda) \mapsto V(\lambda)$ induces an isomorphism from $\mathbb{Z}\left[\Lambda_{+}\right]$to the Grothendieck ring of $A_{2}$. Using induction, it suffices to check that for all $\lambda \in \Lambda_{+}$,

$$
\begin{aligned}
v(0) v(\lambda) & =v(\lambda) \\
v\left(\lambda_{1}\right) v(\lambda) & =v\left(\lambda+\lambda_{1}\right)+v\left(\lambda-\lambda_{1}+\lambda_{2}\right)+v\left(\lambda-\lambda_{2}\right) \\
v\left(\lambda_{2}\right) v(\lambda) & =v\left(\lambda+\lambda_{2}\right)+v\left(\lambda-\lambda_{2}+\lambda_{1}\right)+v\left(\lambda-\lambda_{1}\right),
\end{aligned}
$$

where $v(\lambda)$ is defined as 0 for $\lambda \notin \Lambda_{+}$, because similar relations hold in the Grothendieck ring. (Note that we cannot argue from an a priori hypothesis that $\mathbb{Z}\left[\Lambda_{+}\right]$is the Grothendieck ring of a category, because such a construction assumes the existence of internal clasps, which in turn depends on Theorem 6.1.) In terms of webs, the first relation states that if $c$ and $d$ are two segregated clasps, then $B(c d)$ is empty unless $c=d^{*}$, in which case it has one element. This follows from Lemma 6.5 or from arguments similar to those of Lemma 6.8. Similarly, the other relations are equivalent to Lemma 6.8.

\subsection{The $B_{2}$ and $G_{2}$ cases.}

Theorem 6.9. Let $C$ be a $B_{2}$ clasp sequence and let wt $C$ be the corresponding sequence of weights. Then the vector spaces $W(C)$ and $\operatorname{Inv}(V($ wt $C))$ have the same dimension.

Theorem 6.10. Let $C$ be an $G_{2}$ clasp sequence and let $\mathrm{wt} C$ be the corresponding sequence of weights. Then the vector spaces $W(C)$ and $\operatorname{Inv}(V($ wt $C))$ have the same dimension.

To establish these two results we mainly need to alter various technical definitions given for the $A_{2}$ case; most of the lemmas leading up to Theorem 6.2 and the proof of the theorem then carry over word-for-word. An $\mathrm{H}$-web in the $B_{2}$ or $G_{2}$ spider is the web:

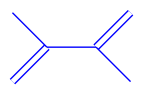

In both the $B_{2}$ and $G_{2}$ spiders, one can define an H-move on a basis web $w$ with two adjacent endpoints labelled 1 and 2. An H-move at two such endpoints consists of attaching an $\mathrm{H}$-web to $w$, provided that this operation does not create an elliptic face, and then replacing an internal double edge by a tetravalent vertex in the $B_{2}$ spider or by a perpendicular single edge in the $G_{2}$ spider:

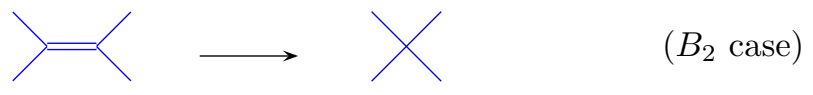




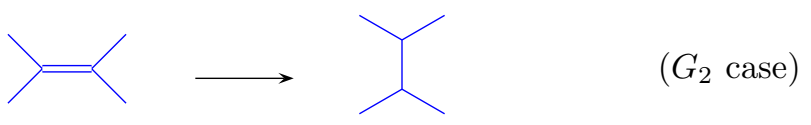

If attaching an $\mathrm{H}$-web would result in an elliptic face, then there are two alternatives: Either an $\mathrm{H}$-web can be removed (possibly after introducing an internal type 2 strand by the above operations), or the two endpoints make a "Y":

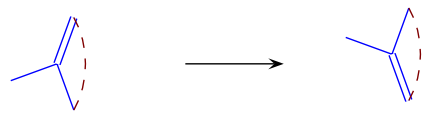

or, in the $G_{2}$ case, they make an "H" with only one type 2 strand:

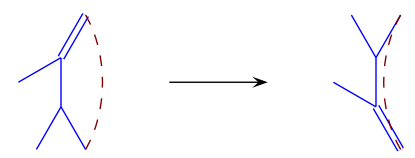

In each of these exceptional cases, the operation indicated is the H-move.

The operation of cutting along a minimal path is slightly more complicated than before, because the path might cut diagonally across a vertex in the $B_{2}$ spider or contain a type 1 edge in the $G_{2}$ spider. The operation is defined by first introducing a type 2 edge, as is sometimes also necessary for an $\mathrm{H}$-move, by reversing the contraction operation of Figure (7). A core is defined in the same way for all three rank 2 spiders. The stair-step construction is essentially the same as before. Each square is an $\mathrm{H}$-web, with the result that sewing together two cores with stair steps in between results in many internal type 2 strands, which are removed by the operation of Figure (7).

The biggest difference between the three rank 2 cases is in the statement and proof of the analogues of Lemma 6.8. We wish to check that, if $s \in\{1,2\}$ and $c$ and $d$ are arbitrary clasps of weight $\lambda$ and $\mu$, then

$$
|B([s] c d)|=\operatorname{dim} \operatorname{Inv}\left(V_{s} \otimes V(\lambda) \otimes V(\mu)\right) .
$$

For this purpose, one would like to argue that an element of $B([s] c d)$ has no hyperbolic faces. As before, it is convenient to consider segregated clasps, where here a clasp is segregated if it is of the form $11 \ldots 122 \ldots 2$, as well as reverse segregated clasps of the form $22 \ldots 211 \ldots 1$. In the $B_{2}$ case, it is easy to enumerate the elements of $B([1] c d)$ if $c$ is segregated and $d$ is reverse segregated, as well as the elements of $B([2] c d)$ if $c$ is reverse segregated and $d$ is segregated. A web $w$ in such a basis web set cannot have negative curvature, and after removing bare strands, it is either empty or its the shape of its boundary is one of the following (possibly with $c$ and $d$ switched):
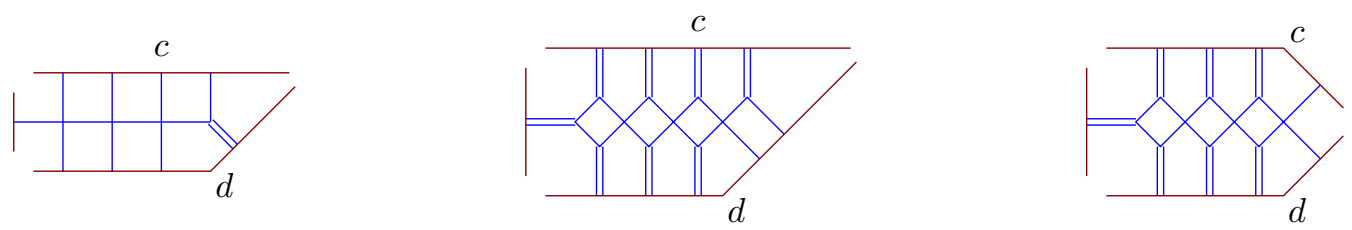

In the $G_{2}$ case, the computation is again simpler if one of $c$ and $d$ is segregated and the other is reverse segregated. No webs in $B([1] c d)$ have any negative curvature, but a web in $B([2] c d)$ might have one negatively curved face, namely one with three type 2 strands and one type 1 strand incident to it:

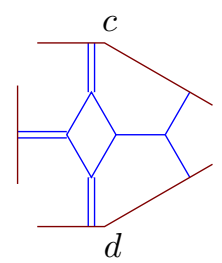

All other $G_{2}$ webs in $B([2] c d)$ are flat. An exhaustive enumeration of such webs in both spiders verifies equation (8). The details are left to the reader. 
Finally, in the $G_{2}$ spider, unlike in the $A_{2}$ and $B_{2}$ spiders, web with a partly convex clasp does not necessarily vanish in the clasped web space $W\left(\left[s_{1}\right]\left[s_{2}\right] \ldots\left[s_{n}\right]\right)$. Recall that the clasped web space is defined as the subspace of the unclasped web space $W\left(s_{1} s_{2} \ldots s_{n}\right)$ spanned by non-elliptic webs with non-convex clasps and no internal type 2 edges, and that there is also a kernel $I\left(\left[s_{1}\right]\left[s_{2}\right] \ldots\left[s_{n}\right]\right)$ spanned by webs $w$ such that there is a cut path transverse to all edges of $w$ which cuts off a clasp of lower weight. In order to show that the $G_{2}$ spider is well-defined, we need the following lemma:

Lemma 6.11. The spaces $I\left(\left[s_{1}\right]\left[s_{2}\right] \ldots\left[s_{n}\right]\right)$ and $W\left(\left[s_{1}\right]\left[s_{2}\right] \ldots\left[s_{n}\right]\right)$ are transverse in $W\left(s_{1} s_{2} \ldots s_{n}\right)$.

Proof: Rank the webs in $W\left(s_{1} s_{2} \ldots s_{n}\right)$ first by the number of vertices, and second by the sum of the weights of minimal cut paths cutting off each string $s_{i}$. We consider a change of basis which is lowertriangular with respect to this ranking: For each web $w \in W\left(s_{1} s_{2} \ldots s_{n}\right)$, choose a minimal cut path $p_{i}$ cutting off each $s_{i}$ as close as possible to the boundary. The cut paths are unique by the $G_{2}$ analogue of Lemma 6.6, and they cannot cross, although they could in principle share segments. Recall the equation for a double edge from the equations (4), rearranged slightly:

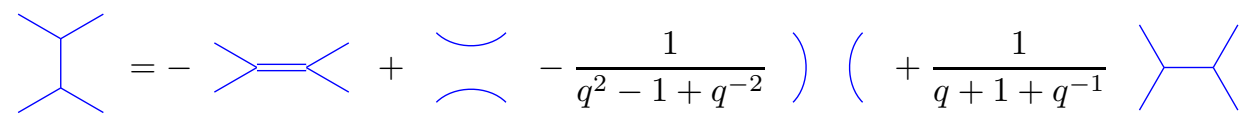

Applying this equation, we can convert every single edge contained in a path $p_{i}$ to a transverse double edge; the other terms are all lower with respect to the ranking of webs. This produces a new basis for $W\left(s_{1} s_{2} \ldots s_{n}\right)$ in which $I\left(\left[s_{1}\right]\left[s_{2}\right] \ldots\left[s_{n}\right]\right)$ and $W\left(\left[s_{1}\right]\left[s_{2}\right] \ldots\left[s_{n}\right]\right)$ are manifestly complements.

\section{EXPLICIT FORMULAS FOR $A_{2}$ CLASPS}

Although internal clasps must exist by Section 5, the argument given there is too indirect for practical computations. In this section, we give an explicit formulas for $A_{2}$ clasps. Note first that clasps are sent to each other by composition with $H$ webs:
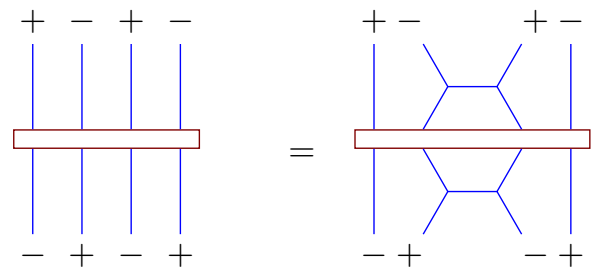

Thus, it suffices to derive a formula for segregated clasps.

Lemma 7.1. If $s$ is a segregated sign string and $t^{*}$ is another sign string of lower or incomparable weight, then a basis web $w \in B(s t)$ has either a " $U$ " or a " $Y$ " attached to $s$.

Proof: The proof is also similar to that of Lemma 6.5. We assume the minimal counterexample. First, the web $w$ must be connected, for if a connected component meets $t$ but not $s$, it may be discarded to produce a smaller counterexample; if it meets $s$ but not $t$, then it is a smaller counterexample; and if all components meet both $s$ and $t$, then one of them must be a smaller counterexample. Second, there can be no "Y" or "H" attached at $t$, for otherwise they may be discarded to produce a smaller counterexample. (Note that a "U" attached to $t$ has already been eliminated.)

Since $w$ is non-elliptic, the total exterior curvature is at least $360^{\circ}$. Since there is no "U", "Y", or "H" attached at $t^{*}$, the total curvature along $t$ is at most $0^{\circ}$. Moreover, unless $w$ is a bare strand (which is not a counterexample), the two arcs connecting $s$ to $t$ have curvature at most $120^{\circ}$. By the hypothesis that $s$ has no "Y", the curvature in each segregated segment of $S$ is at most $0^{\circ}$ also. Finally, since $s$ has no "U", the curvature at the single arc connecting opposite signs of $s$ is at most $60^{\circ}$, for a total of at most $300^{\circ}$, a 
contradiction:

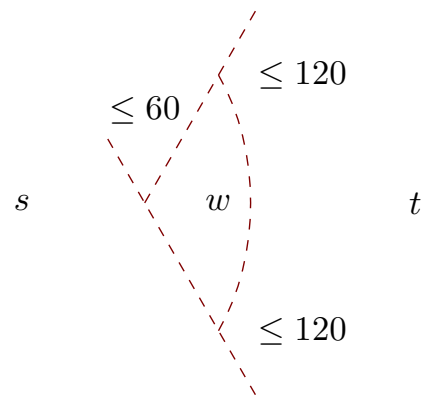

By Lemma 7.1, if a web $w \in W\left(s s^{*}\right)$ annihilates any "U" or "Y", it satisfies the annihilation axiom of a clasp. Moreover, among terms in $w$, only $\beta_{s}$ (the web consisting solely of parallel strands) has the weight of $s$ as its cut weight. Therefore all other terms are annihilated by such $w$, and the annihilation property implies that $w$ is an idempotent under concatenation if the coefficient of the leading term $\beta_{s}$ is 1 . Given these facts, a routine argument by induction (see Wenzl 24 and Ohtsuki and Yamada |16]) establishes that

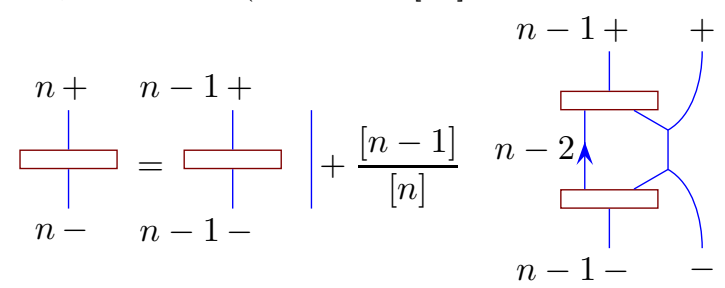

recursively defines a clasp of weight $n \lambda_{1}$, and a computation shows that

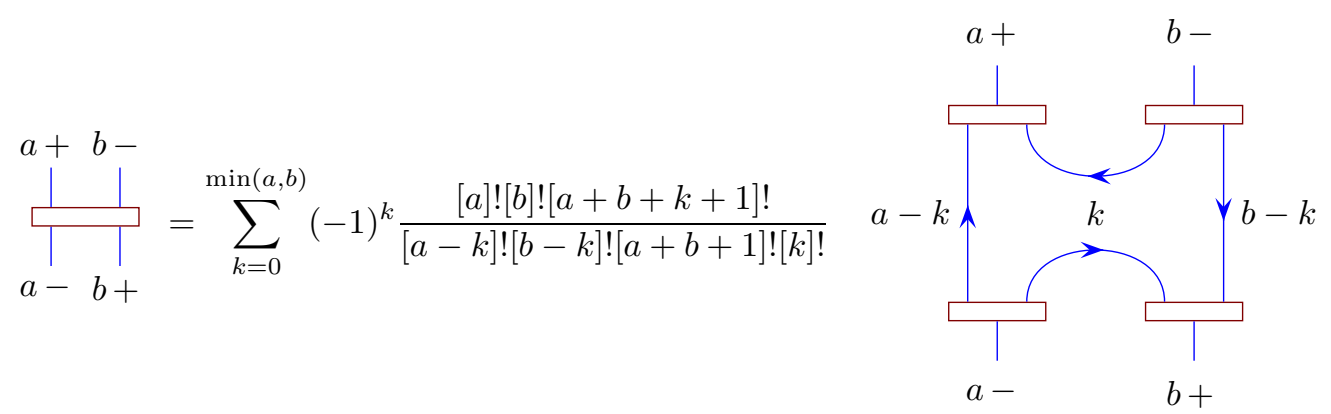

defines a segregated clasp of weight $a \lambda_{1}+b \lambda_{2}$.

\section{Applications AND PROBlems}

8.1. Higher rank. The main open problem related to the combinatorial rank 2 spiders is how to generalize them to higher rank. A proper generalization would consist of a complete set of generators and relations for the higher-rank spiders; the strand sets would correspond to the fundamental representations and their tensor products. It is easy to make a HOMFLY spider which corresponds to the HOMFLY polynomial, but this spider describes the invariant theory of $A_{n}$ only in the stable limit of large $n$; the $A_{n}$ web spaces for any fixed $n$ are quotients of the HOMFLY web spaces. (Recently, Murakami, Ohtsuki, and Yamada [15 have defined the HOMFLY spider in terms of trivalent graphs; this is a step toward an explicit description of the unstable truncation.) The generalization to a higher-rank Lie algebra $\mathfrak{g}$, if it exists, would also likely involve formal angles related to the Coxeter geometry of $\mathfrak{g}$; note that in both the $A_{2}$ and $B_{2}$ spiders, a trivalent vertex is dual to a Weyl alcove. Note also that in all three rank 2 spiders, a large, flat basis web (one with neither elliptic nor hyperbolic faces) coincides with the Voronoi tiling of the plane given by the weight lattice of the corresponding Lie algebra.

The bases given by the combinatorial rank 1 spider are dual to Lusztig's canonical bases [2]. Those of the rank 2 spiders are almost certainly also dual to canonical bases or are closely related, because canonical bases have the same symmetry of cyclic permutation of tensor factors, and because of the integrality and positivity properties of the coefficients in equations (2), (3), and (4). 
8.2. Generalized $6 j$ symbols. Given four webs $w_{1}, w_{2}, w_{3}$, and $w_{4}$ and six clasps $c_{12}, c_{13}, c_{14}, c_{23}, c_{24}$, and $c_{34}$ in some spider, their tetrahedron symbol is defined as the value of the compound web

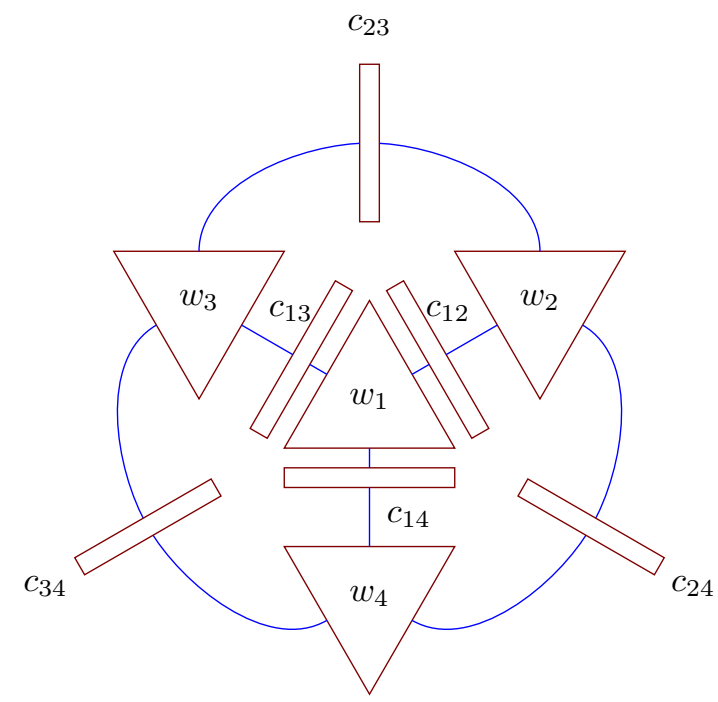

provided that the four webs $w_{i}$ are members of the appropriate clasped web spaces. The tetrahedron symbol is closely related to the $6 j$ symbol, which expresses the change of basis from

$$
\bigoplus_{c_{14}} W\left(c_{12} c_{13} c_{14}\right) \otimes W\left(c_{43} c_{42} c_{41}\right)
$$

to

$$
\bigoplus_{c_{23}} W\left(c_{42} c_{12} c_{32}\right) \otimes W\left(c_{13} c_{43} c_{23}\right)
$$

via the identification of both with $W\left(c_{12} c_{13} c_{43} c_{42}\right)$, where in general $c_{i j}=c_{j i}^{*}$. Up to normalization, the $A_{1}$ tetrahedron symbol at $q=1$ equals the Racah-Wigner $6 j$ symbol used in mathematical physics [14. Using the $A_{1}$ spider, Masbaum and Vogel have found a new proof of the Racah formula for the $6 j$ symbol and its quantum generalization [12]. The combinatorial rank 2 spiders could be equally useful for understanding the rank 2 generalization of the $6 j$ symbol.

8.3. Practical computation of rank 2 link invariants. If one is interested in computing link invariants, equations (2), (3), and (4) can be interpreted as inductive rules for evaluating links and knotted graphs without boundary in the web space $W(\emptyset)$. Mollard [13] and Sinha [19] have independently written computer implementations of this algorithm for $G_{2}$, and there are well-known computer programs to compute the Jones, HOMFLY, and Kauffman polynomials using the same basic strategy.

Spiders suggest an alternative method for computing the same invariants which is more efficient than a direct application of the above rules to closed links and graphs. The method consists of assembling a link projection, as a web, from indiviual crossings using spider operations, and reducing intermediate webs to linear combinations of basis webs. For example, to evaluate the quantum $A_{1}$ link invariant (the Jones polynomial) of a figure eight knot, we can decompose the knot as a nested sequence of three tangles $a, b$, 
and $c$ :

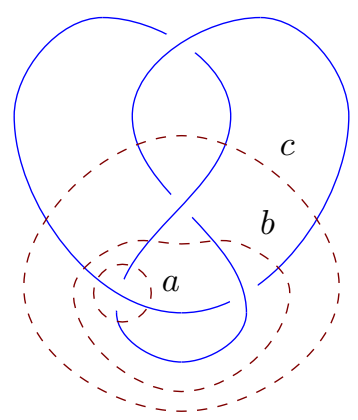

We recall some basic identities in the $A_{1}$ spider:

$$
\begin{aligned}
& \bigcirc=-q^{1 / 2}-q^{-1 / 2} \\
& \left.Y=-q^{1 / 4}\right)\left(-q^{-1 / 4} \square\right. \\
& \left.\bigcirc=q^{3 / 4}\right)
\end{aligned}
$$

The tangle $a$ is a right-handed crossing. Its expansion leads to an expansion of the tangle $b$ :

$$
Y\left(=-q^{1 / 4}\right) \bigcirc\left\langle-q^{-1 / 4} \backslash /=(1-q)\right)\left(+q^{-1 / 2} \circlearrowright\right.
$$

which leads to an expansion of tangle $c$ :

$$
\overbrace{\backslash}=(1-q) \backslash / \backslash q^{-1 / 2} \circlearrowright=\left(q^{5 / 4}-q^{1 / 4}\right))\left(+\left(q^{3 / 4}-q^{-1 / 4}+q^{-5 / 4}\right)\right.
$$

which leads to the evaluation of the entire knot projection:

$$
\begin{aligned}
& =\left(q^{5 / 4}-q^{1 / 4}\right) \bigcirc \bigcirc+\left(q^{3 / 4}-q^{-1 / 4}+q^{-5 / 4}\right) \bigcirc O \\
& =-\left(q^{1 / 2}-q^{-1 / 2}\right)\left(q^{2}-q+1-q^{-1}+q^{-2}\right)
\end{aligned}
$$


Given a knot $K$ and a clasp $c$ in one of the combinatorial spiders presented here, consider the clasped cabling of $K$. I.e., replace the strand of $K$ by several strands tied together with $c$, for example:
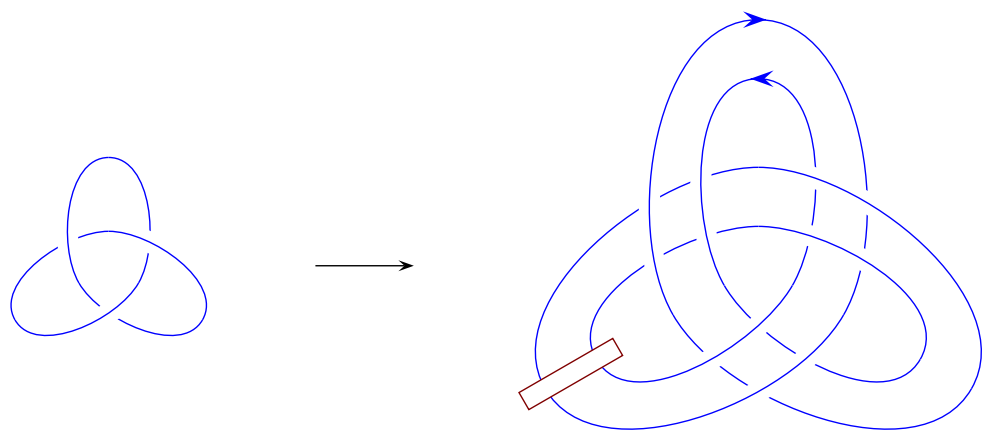

The value of a web such as the one in Figure (9) is also a regular isotopy invariant of $K$; it is the ReshetikhinTuraev ribbon graph invariant of $K$ colored by an irreducible representation whose weight is the highest weight of $c$ [17. By the isomorphism between combinatorial and algebraic spiders, clasps are a complete set in the sense that these clasped invariants yield all of the information about $K$ that can be obtained from applying the unclasped link invariants to all cablings of of $K$ and all other satellites of $K$. Moreover, the value of a clasped cabling of $K$ can be computed more easily than an unclasped cabling, because clasped web spaces are much smaller vector spaces than their unclasped counterparts.

8.4. Combinatorial consequences. Although Theorems 6.1.6.9, and 6.10 are properly results in representation theory, they are also interesting as results in enumerative combinatorics. John Stembridge and Richard Stanley [20] noted that a $B_{2}$ basis web $w \in B(11 \ldots 1)$ is equivalent to a matching of $2 n$ cyclically ordered points with no 6 -point star, meaning that among the $2 n$ points, there are no six in cyclic order no six points $p_{1}, p_{2}, p_{3}, q_{1}, q_{2}, q_{3}$ with each $p_{i}$ matched to $q_{i}$. On other hand, the number of such matchings with no $2 k$-point star is known to be $\operatorname{dim} \operatorname{Inv}\left(V^{\otimes 2 n}\right)$, where $V$ is the defining representation of $\operatorname{sp}(2 k-2)$ [21]. Thus, this special case of Theorem 6.9 was known previously.

It is interesting that ordinary trivalent graphs are related to the exceptional Lie algebra $G_{2}$. One corollary of this surprising connection is the following enumerative result:

Theorem 8.1. For $n \geq 3$, let $a_{n}$ be the number of triangulations of a fixed convex $n$-gon such that at least six triangles meet at each internal vertex, and let

$$
A(x)=1+x^{2}+\sum_{n} a_{n} x^{n}
$$

be a generating function. For $n \geq 0$, let

$$
b_{n}=\operatorname{dim} \operatorname{Inv}_{G_{2}}\left(V\left(\lambda_{1}\right)^{\otimes n}\right)
$$

for $n \geq 0$, and let

$$
B(x)=\sum_{n} b_{n} x^{n}
$$

be a generating function. Then

$$
B(x)=A(x B(x))
$$

Proof: (Sketch) By Theorem 6.10, $b_{n}$ may equally well be defined as the number of $G_{2}$ basis webs with $n$ endpoints. Each triangulation is dual to a connected basis web. Equation 8.1) becomes a standard relation between the number of connected graphs of a certain type and the number of disjoint unions of such graphs. More precisely, consider $n$ boundary points on a disk with one marked, and consider a basis web $w \in B(11 \ldots 1)$ with this boundary. Then $w$ is given by an ordered tree whose vertices are its connected components: The root is the component with the marked vertex, and the children of each component are 
the adjacent components other than the parent, if there is one. The children of each component are ordered going counterclockwise, with the distiguished boundary point separating the first and last children of the root. Equation (8.1) is the usual generating function for ordered trees [3, p. 11-12].

The sequence $\left\{b_{n}\right\}$ is easy to compute using character theory. Thus, Theorem 8.1 produces a fast algorithm for computing $\left\{a_{n}\right\}$.

It is easy to show that the radius of convergence of $B(x)$ is $1 / 7$. Then by Theorem 8.1, the radius of convergence of $A(x)$ is at least $B(1 / 7) / 7$. Furthermore, numerical evidence supports the conjecture that it is exactly $B(1 / 7) / 7$, i.e., that

Conjecture 8.2. If $a_{n}$ and $b_{n}$ are defined as in Theorem 8.1, then

$$
\lim _{n \rightarrow \infty} \sqrt[n]{a_{n}}=\frac{7}{\sum_{n=0}^{\infty} b_{n} 7^{-n}}=6.811 \ldots
$$

Our equinumeration theorems count all basis webs with a fixed boundary. However, for each $n$ and $k$, one can also consider the set of $G_{2}$ basis webs, for example, with $n$ endpoints of type 1 and $k$ internal vertices. The role of these sets and their cardinality in representation theory is not known.

Given an $A_{2}$ basis-web set $B(s)$ for some sign string $s$, there are various H-maps corresponding to different adjacent pairs of signs. But if $s^{\prime}$ is another sign string of the same weight as $s$, then any two sequences of H-maps permuting $s$ into $s^{\prime}$ yield the same bijection $B(s) \rightarrow B\left(s^{\prime}\right)$, provided that the first and the last sign of $s$ are not considered adjacent. On the other hand, if a sign is moved all the way around the boundary by H-maps, holonomy arises:

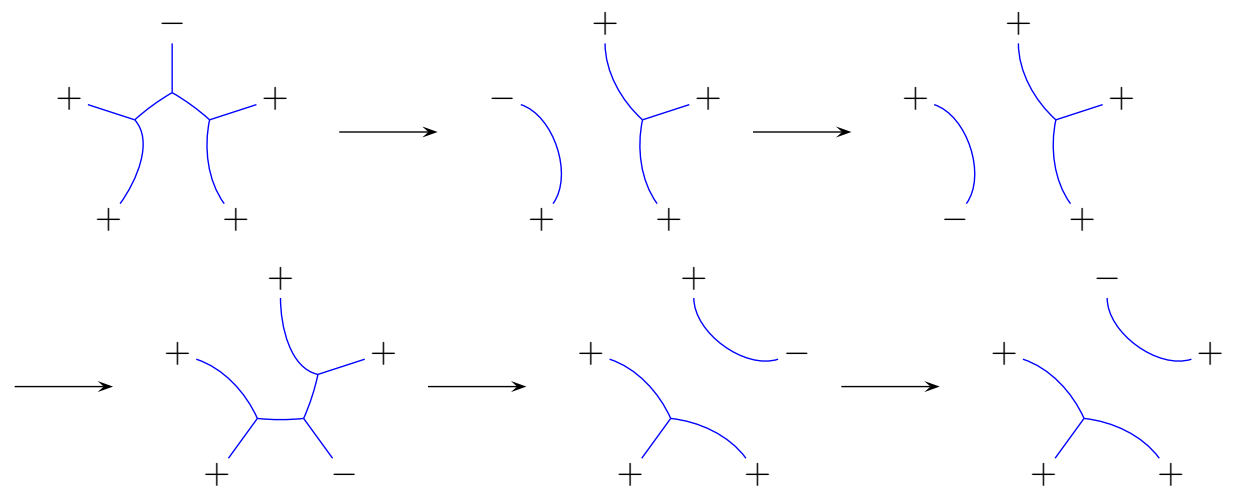

It would be interesting to compute the cycle structure or establish properties of this holonomy, which is related to a linear action of the braid group on

$$
\operatorname{Inv}\left(\left(V_{+} \oplus V_{-}\right)^{\otimes n}\right) \cong \bigoplus_{s} W(s)
$$

where the direct sum on the right is taken over all sign strings of length $n$.

\section{REFERENCES}

[1] J. W. Barrett and B. W. Westbury. Spherical categories. hep-th preprint \#9310164.

[2] I. Frenkel and M. Khovanov. Canonical bases in tensor products and graphical caluclus for $U_{q}\left(\mathfrak{s l}_{2}\right)$. Preprint, 1995.

[3] I. P. Goulden and D. M. Jackson. Combinatorial Enumeration. Wiley, New York, 1983.

[4] F. Jaeger. Confluent reductions of cubic plane maps. In Graph Theory and Combinatorics International Conference, Marseille, 1990.

[5] V. F. R. Jones. Index of subfactors. Invent. Math., 72:1-25, 1987.

[6] L. H. Kauffman. State models and the Jones polynomial. Topology, 26:395-407, 1987.

[7] L. H. Kauffman and S. L. Lins. Temperley-Lieb recoupling theory and invariants of 3-manifolds. Annals of mathematics studies. Princeton University Press, Princeton, N. J., 1994.

[8] A. A. Kirillov. Elements of the Theory of Representations. Springer-Verlag, Berlin, 1976.

[9] G. Kuperberg. The quantum $G_{2}$ link invariant. Int. J. Math., 5:61-85, 1994.

[10] W. B. R. Lickorish. Calculations with the Temperley-Lieb algebra. Comment. Math. Helv., 67:571-591, 1992.

[11] G. Lusztig. Canonical bases arising from quantized enveloping algebras. J. Amer. Math. Soc., 3:447-498, 1990.

[12] G. Masbaum and P. Vogel. 3-valent graphs and the Kauffman bracket. Pacific J. Math., 164:361-381, 1994.

[13] M. Mollard, 1992. personal communication.

[14] G. Moore and N. Seiberg. Classical and quantum conformal field theory. Comm. Math. Phys., 123:177-254, 1989.

[15] H. Murakami, T. Ohtsuki, and S. Yamada. Homfly polynomial via an invariant of colored planar graphs. Preprint, 1995. 
[16] T. Ohtsuki and S. Yamada. Quantum su(3) invariants via linear skein theory. Preprint, 1995.

[17] N. Yu. Reshetikhin and V. G. Turaev. Ribbon graphs and their invariants derived from quantum groups. Comm. Math. Phys., 127:1-26, 1990.

[18] G. Rumer, E. Teller, and H. Weyl. Eine für die Valenztheorie geeignete Basis der binaren Vektorinvarianten. Nachr. Ges. Wiss. gottingen Math.-Phys. Kl., pages 499-504, 1932.

[19] D. Sinha, 1992. personal communication.

[20] R. P. Stanley and J. R. Stembridge, 1991. personal communication.

[21] S. Sundaram. Tableaux in the representation theory of the classical Lie groups. In Invariant theory and tableaux (Minneapolis, MN, 1988), volume 19 of IMA Vol. Math. Appl., pages 191-225. Springer, New York, 1990.

[22] H. N. V. Temperley and E. H. Lieb. Relations between the 'percolation' and 'coloring' problem and other graph-theoretical problems associated with regular planar lattices: some exact reults for the 'percolation' problem. Proc. Roy. Soc. London, Ser. A, 322:251-280, 1971.

[23] T. Van Zandt. PSTricks: PostScript macros for generic TEX. Available at ftp://ftp.princeton.edu/pub/tvz .

[24] H. Wenzl. On sequences of projections. In C. R. Math. Rep. Acad. Sci. Canada IX, pages 5-9, 1987.

Department of Mathematics, Yale University, New Haven, Ct 06520

E-mail address: greg@math.yale.edu 